\title{
Computing absolute and essential spectra using continuation
}

\author{
Jens D.M. Rademacher ${ }^{\mathrm{a}, *}$, Björn Sandstede $^{\mathrm{b}}$, Arnd Scheel $^{\mathrm{c}}$ \\ ${ }^{a}$ Centre for Mathematics and Computer Science (CWI), Kruislaan 413, 1098 SJ Amsterdam, The Netherlands \\ ${ }^{\mathrm{b}}$ Department of Mathematics, University of Surrey, Guildford, GU2 7XH, UK \\ ${ }^{c}$ School of Mathematics, University of Minnesota, Minneapolis, MN 55455, USA
}

Received 5 October 2006; received in revised form 7 February 2007; accepted 24 March 2007

Available online 31 March 2007

Communicated by Y. Nishiura

\begin{abstract}
A continuation approach to the computation of essential and absolute spectra of differential operators on the real line is presented. The advantages of this approach, compared with direct eigenvalue computations for the discretized operator, are the efficient and accurate computation of selected parts of the spectrum (typically those near the imaginary axis) and the option to compute nonlinear travelling waves and selected eigenvalues or other stability indicators simultaneously in order to locate accurately the onset to instability. We also discuss the implementation and usage of this approach with the software package AUTO and provide example computations for the FitzHugh-Nagumo and the complex Ginzburg-Landau equation.
\end{abstract}

(C) 2007 Elsevier B.V. All rights reserved.

Keywords: Spectral stability; Continuation; Absolute spectrum; Instability thresholds; Reaction-diffusion systems

\section{Introduction}

Phase transitions in dissipative spatially extended systems often go hand in hand with instabilities of coherent structures. Famous examples are pipe flows, thermal convection, and the transition to chemical turbulence. On the theoretical side, phase transitions in the complex Ginzburg-Landau equation are among the best understood theoretical examples for the role of instabilities in dissipative non-equilibrium systems, and yet there are still many unresolved challenges in this "simple" model.

Identifying the onset of instability can present major computational challenges due to the many active degrees of freedom that systems in large domains exhibit. An additional difficulty arises when convective transport mechanisms cause linearized operators to be non-normal, leading to ill-conditioned eigenvalue problems where pseudo-spectra become relevant [18], and to sensitive dependence on boundary

\footnotetext{
* Corresponding address: Centre for Mathematics and Computer Science (CWI), Modelling Analysis and Simulation, Kruislaan 413, 1098 SJ Amsterdam, The Netherlands.

E-mail address: rademach@cwi.nl (J.D.M. Rademacher).
}

conditions. One is then led to distinguish different types of instability: At the onset of convective instability, perturbations of a coherent structure grow in norm but decay at each fixed point in space, and it is only at the subsequent onset of an absolute instability that perturbations grow at each fixed point in physical space.

Beyond this idealized characterization in an unbounded domain, it is often of interest to determine the onset of instability in a large but bounded domain, with boundaries formed by either physical boundaries or domain walls in the pattern. In [13], we showed that the stability of coherent structures in such large but finite domains can be characterized using spectral information on the unbounded domain together with information on localized boundary modes: As the size of the domain goes to infinity, spectra in large domains converge to a limiting set which is the disjoint union of a continuous part formed by curves and a discrete part that consists of localized modes and resonance poles. In the case of periodic boundary conditions, the continuous part is given by the essential spectrum of the prevailing background pattern. In the more typical case of separated boundary conditions, it is given by the absolute spectrum of this background state. Eigenvalues of the linearization in finite-size domains accumulate at these 
spectral curves as the domain diameter goes to infinity. The location of these curves is independent of the number and location of defects embedded in the regular pattern and of the imposed boundary conditions.

Our goal in this paper is to provide efficient and accurate numerical procedures to determine the continuous part of the limiting spectrum. We will outline algorithms that are based on complex extensions of Fourier and Laplace transforms for spatio-temporally homogeneous background patterns, and on Floquet-Bloch theory for spatio-temporally periodic background states. Knowing the location of all accumulation points of eigenvalues greatly simplifies the task of computing the onset of instability: On the one hand, the convergence of eigenvalues away from the limiting curves is exponential in the domain size, so that accurate information can be gained by computations on only moderately sized domains. On the other hand, the knowledge of the limiting curves makes it possible to construct Cayley transforms in order to either avoid these eigenvalue clusters on large domains and to resolve only isolated eigenvalues and resonance poles, or else to efficiently compute eigenvalues with maximal real part near the boundaries of these eigenvalue clusters.

We emphasize that, in many problems, the direct computation of spectra on the large domain is either intractable or at least highly ill-conditioned. While computations of pseudo-spectra often remedy some of these problems, essential and absolute spectra provide valuable additional information, which is, moreover, independent of the artificial cut-off choice for the norm of the resolvent, the size of the domain, and the particular boundary conditions.

The methods that we describe here have already been used to compute the onset of instability of coherent structures in reaction-diffusion systems, for instance, in the computation of the onset of spiral breakup in $[19,12]$ and in the numerical stability proof for wide pulses in a model for $\mathrm{CO}$ oxidation on $\operatorname{Pt}(110)$ surfaces [15]. We shall now outline these two applications in more detail: The numerical computation of absolute spectra was exploited for the direct computation of linearized spectra of spiral waves in large disks: In [12], the absolute spectrum of these two-dimensional vortex-like structures was computed using only the spectral properties of the emitted wave trains, utilizing the continuation algorithm that we describe in this article. The results were then exploited in [19] to construct suitable Cayley transforms for the direct computation of spectra of the elliptic linearized operator on the large disk using subspace iterations. The large-scale spectral computations in [19] are in excellent agreement with the predictions of the continuation algorithm in [12]; see [19,

Figure 7]. In the second example, the stability of pulses with a wide plateau, which are obtained by gluing together widely separated fronts and backs, was determined from the computation of the absolute spectrum of the plateau state [15]. Direct computations of spectra in large one-dimensional domains delivered only ambiguous results which depended heavily on the choice of boundary conditions and the relative size of plateau versus background state $[20,15]$.
One of the examples in this paper follows up on the spiralwave computations by computing absolute stability boundaries of plane waves in the complex Ginzburg-Landau equation which arise as far-field patterns of vortices. We show that wave trains may destabilize due to robust persistent reflection at generic boundaries on finite domains, while wave trains remain stable on the unbounded domain. In other words, there are parameter regions where perturbations of wave trains decay pointwise on the unbounded domain, but grow in norm on any finite domain for almost any boundary condition. The latter parameter regime provides a prominent example that pointwise stability does not guarantee stability in large but finite domains. In particular, the crossing of double roots of the dispersion relation together with the pinching condition that characterizes the onset of absolute instability in unbounded domains [1,17] is only a sufficient, yet not necessary, criterion for the onset of instability in finite-size domains.

Technically, the present article considers reaction-diffusion patterns on large intervals as a prototype of spatially extended dissipative dynamical systems. The methods however are readily adapted to much more general problems. Typically, the method assumes that the linearized problem can be written as a family of Fredholm operator equations $\mathcal{L}(\lambda, v) w=0$ where $w$ lives in a space of functions on a bounded (not large) domain. The equation $\mathcal{L}(\lambda, v) w=0$ is obtained upon substituting the spatio-temporal Floquet-Bloch ansatz $u(t, x)=\exp (\lambda t+$ $v x) w(t, x)$ into the original linearized problem, where the temporal frequency $\omega=-i \lambda$ and the spatial wavenumber $k=-\mathrm{i} v$ are typically complex. The continuation procedure outlined below can be readily adapted to this type of problem, e.g., the Kuramoto-Sivashinsky equation [11]. We give details for the implementation for one-dimensional boundary-value problems using the continuation software AUTO. Alternatively, the package TRILINOS [7] can be used which is optimized for large-scale systems. Problems where inverting $\mathcal{L}$ involves the solution of boundary-value problems on multi-dimensional domains can sometimes be approached using finite-dimensional Galerkin approximations in the transverse components.

To set the scene, and to further explain the significance of absolute and essential spectra, we review the most straightforward situation in which they arise. Consider reaction-diffusion systems

$u_{t}=D u_{x x}+c u_{x}+f(u), \quad x \in \mathbb{R}$

where $u \in \mathbb{R}^{N}$ and $f$ is smooth. To ensure that (1.1) is wellposed, we shall assume for simplicity throughout this paper that $D$ is a positive diagonal matrix. Suppose now that $u_{*}(x)$ is a stationary solution of (1.1) so that

$\left|u_{*}(x)-u_{ \pm}(x)\right| \rightarrow 0 \quad$ as $x \rightarrow \pm \infty$

where the asymptotic states $u_{ \pm}(x)$ are constant or periodic in $x$. Linearizing (1.1) about $u_{*}(x)$, we obtain the operator

$\mathcal{L}_{*}=D \partial_{x x}+c \partial_{x}+\partial_{u} f\left(u_{*}(x)\right)$

whose spectrum decides upon linear stability of the equilibrium $u_{*}$ as a solution of (1.1). 
The spectrum of the operator $(1.2)$ on $L^{2}\left(\mathbb{R}, \mathbb{C}^{N}\right)$ is the disjoint union of the essential spectrum $\Sigma_{\text {ess }}$ and the point spectrum $\Sigma_{\mathrm{pt}}$ which consists by definition of all isolated eigenvalues with finite multiplicity (and is therefore discrete). While the point spectrum involves the full nonlinear wave $u_{*}$, the boundary of the essential spectrum is determined entirely by the linearization of (1.1) about the asymptotic states $u_{ \pm}[6$, Appendix to Section 5].

Also of interest are finite but large domains of the form $(-\ell, \ell)$ with $\ell \gg 1$. When considering (1.2) on $(-\ell, \ell)$, we add appropriate linear separated boundary conditions of the form

$$
\begin{aligned}
Q_{ \pm}^{\mathrm{bc}} & \left(\begin{array}{c}
u( \pm \ell) \\
u_{x}( \pm \ell)
\end{array}\right)=0, \quad Q_{ \pm}^{\mathrm{bc}} \in \mathbb{R}^{2 N \times 2 N}, \\
& \operatorname{rank} Q_{ \pm}^{\mathrm{bc}}=N
\end{aligned}
$$

at $x= \pm \ell$. While the spectrum $\Sigma_{\ell}$ of the operator $\mathcal{L}_{*}$ on $(-\ell, \ell)$ is then necessarily discrete for each finite $\ell$, we can still distinguish different parts in the limit $\ell \rightarrow \infty$ [13]: Generically, the set $\Sigma_{\ell}$ converges in the symmetric Hausdorff distance to a limiting set $\Sigma_{\infty}$ which again consists of a discrete and a continuous part. The discrete part is the union of the extended point spectrum $\Sigma_{\text {ext }}$, which contains in particular the point spectrum $\Sigma_{\mathrm{pt}}$ of the profile $u_{*}(x)$ on $\mathbb{R}$, and the boundary spectrum $\Sigma_{\mathrm{bc}}$, which is generated by the boundary conditions (1.3). The continuous part is called the absolute spectrum which, in general, differs from the essential spectrum. As $\ell \rightarrow$ $\infty$, each element of the absolute spectrum is approached by infinitely many eigenvalues of $\mathcal{L}_{*}$ which therefore cluster near the absolute spectrum. As already alluded to, the discrete part depends on the full profile $u_{*}$ and on the specific boundary conditions employed. The absolute spectrum, however, depends again only on the asymptotic states $u_{ \pm}$, but not on the boundary conditions (as long as they are separated) or the profile $u_{*}$ [14].

In summary, the continuous parts of the spectrum of $\mathcal{L}_{*}$ on $\mathbb{R}$ or $(-\ell, \ell)$ are determined by the asymptotic states $u_{ \pm}$which, we assumed, are constant or periodic in $x$. From now on, we shall therefore focus exclusively on operators with constant or periodic coefficients.

The aim of this paper is to outline reliable and efficient ways to compute these spectra using cheap but accurate continuation algorithms without solving discretized matrix eigenvalue problems on large domains. For the convenience of the reader, we describe in some detail how our strategies can be implemented in the boundary-value solver AUTO [4]. We will also mention various extensions to compute linear spreading speeds and linear instability thresholds such as the boundary of Eckhaus instabilities.

As mentioned above, the methods presented here can be adapted to more general problems. As a general rule, the continuation procedures should generally be applicable to stability analyses of coherent structures in extended systems, including dissipative systems as well as conservative or dispersive equations such as members of the Korteweg-de Vries family or coupled nonlinear Schrödinger equations, or higher order problems such as the Kuramoto-Sivashinsky equation. Some of our arguments can also be adapted to periodic structures in higher space dimensions via Fourier- and Bloch-wave decomposition. We remark, however, that absolute spectra have not been used so far for genuinely higher-dimensional problems.

\section{Notation and hypotheses}

We consider the operator

$\mathcal{L}:=D \partial_{x x}+c \partial_{x}+a(x)$

where we shall always assume that the entries $d_{j}$ of the diagonal matrix $D=\operatorname{diag}\left(d_{j}\right)$ are strictly positive for all $j$ and that the coefficient matrix $a(x)$ is either constant or periodic in $x$ :

Hypothesis (C). The coefficients $a(x)=a_{0} \in \mathbb{R}^{N \times N}$ do not depend on $x$.

Hypothesis (P). The coefficients $a(x) \in C^{1}\left(\mathbb{R}, \mathbb{R}^{N \times N}\right)$ are periodic in $x$ with minimal period $L>0$.

Unless explicitly stated otherwise, we shall always consider the operator $\mathcal{L}$ on $\mathbb{R}$ as a densely defined operator on $L^{2}\left(\mathbb{R}, \mathbb{C}^{N}\right)$ with domain $H^{2}\left(\mathbb{R}, \mathbb{C}^{N}\right)$. The eigenvalue problem

$\mathcal{L} u=D u_{x x}+c u_{x}+a(x) u=\lambda u$

can then also be written as

$u_{x}=v$

$v_{x}=D^{-1}[\lambda u-c v-a(x) u]$

or equivalently as

$U_{x}=[A(x)+\lambda B] U$

where $U=(u, v) \in \mathbb{C}^{2 N}$ and

$A(x)=\left(\begin{array}{cc}0 & 1 \\ -D^{-1} a(x) & -c D^{-1}\end{array}\right), \quad B=\left(\begin{array}{cc}0 & 0 \\ D^{-1} & 0\end{array}\right)$.

Solutions to the eigenvalue problem (2.2) are therefore found by solving the initial-value problem (2.3). For periodic coefficients, we denote by

$$
\begin{aligned}
\Phi_{\lambda}: & \mathbb{C}^{2 N} \longrightarrow \mathbb{C}^{2 N}, \\
\left(u_{0}, v_{0}\right) & \longmapsto \Phi_{\lambda}\left(u_{0}, v_{0}\right):=(u, v)(L)
\end{aligned}
$$

the linear time- $L$ map of (2.3) that associates to each initial condition $\left(u_{0}, v_{0}\right)$ the solution of (2.3) at time $L$. We refer to the eigenvalues of $\Phi_{\lambda}$ as spatial Floquet multipliers and to their logarithms as spatial Floquet exponents.

Note that nonlinear periodic waves can be found as periodic solutions of the first-order system

$U_{x}=F(U, c), \quad F(U, c)=\left(\begin{array}{c}v \\ -D^{-1}[f(u)+c v]\end{array}\right)$

in the variables $U=(u, v) \in \mathbb{R}^{2 N}$. If $u_{*}(x)$ denotes a constant or periodic solution of (2.6), then $a(x)=\partial_{u} f\left(u_{*}(x)\right)$ in (2.2).

Most of the results presented here do not require that $d_{j}>0$ for all $j$. If some of the diffusion coefficients vanish, however, we need that the speed $c$ is non-zero. Alternatively, if a concrete model has $d_{j}=0$ for one or more indices $j$, we may also set 
$d_{j}=\delta$ for those indices with $\delta>0$ sufficiently small. The results in [9, Chapter 3.2] show that the resulting spectra are continuous in $\delta$ as $\delta \rightarrow 0$ on any bounded subset of the complex plane $\mathbb{C}$.

\section{Essential spectra}

\subsection{Characterizing essential spectra via Bloch waves}

For constant coefficients $a(x) \equiv a_{0}$, we consider the Fourier transformed operator

$\mathcal{L}_{v}:=D v^{2}+c v+a_{0}: \quad \mathbb{C}^{N} \rightarrow \mathbb{C}^{N}$

for $v \in \mathbb{C}$. Since the Fourier transform is an isomorphism on $L^{2}\left(\mathbb{R}, \mathbb{C}^{N}\right)$ which turns $\mathcal{L}$ into a multiplication operator, we immediately obtain the following lemma.

Lemma 3.1. For constant coefficients, we have

$\operatorname{spec} \mathcal{L}=\bigcup_{v \in \mathbb{R} R} \operatorname{spec} \mathcal{L}_{\nu}$.

In particular, the following assertions are equivalent:

(i) $\lambda \in \operatorname{spec} \mathcal{L}$;

(ii) $\left[D v^{2}+c v+a_{0}-\lambda\right] u=0$ for some $u \in \mathbb{C}^{N}$ with $u \neq 0$ and some $v \in \mathrm{i} \mathbb{R}$;

(iii) $d(\lambda, v):=\operatorname{det}\left(D v^{2}+c v+a_{0}-\lambda\right)=0$ for some $v \in \mathrm{i} \mathbb{R}$.

While the third condition gives the most compact criterion, the second condition is, in general, preferable for numerical computations.

Note that the spectrum of the matrix $\mathcal{L}_{v}$ consists for each $v$ of precisely $N$ temporal eigenvalues $\lambda_{j}$, counted with multiplicity. Furthermore, the eigenvalues $\lambda_{j}$ can be continued globally in $v$ since they are roots of the complex analytic equation $d(\lambda, \nu)=$ 0 . The essential spectrum is obtained by restricting to purely imaginary $v=\mathrm{i} \gamma$ with $\gamma \in \mathbb{R}$.

Lemma 3.2. For constant coefficients, the essential spectrum of $\mathcal{L}$ on $\mathbb{R}$ has at most $N$ connected components, each containing an eigenvalue $\lambda_{j}$ of the matrix $a_{0}$ (when $\gamma=0$ ) and the point at infinity. Furthermore, we have $|\arg \lambda| \rightarrow \pi$ as $|\gamma| \rightarrow \infty$ in each connected component of the essential spectrum.

We remark that it is not difficult to derive expansions for the location of the curves as $|\gamma| \rightarrow \infty$.

For periodic coefficients $a(x)$, there is a similar characterization. For each $v \in \mathbb{C}$, we define the Bloch-wave operator

$\mathcal{L}_{v}:=D\left(\partial_{x}+v\right)^{2}+c\left(\partial_{x}+v\right)+a(x)$

which is closed and densely defined on $L_{\mathrm{per}}^{2}(0, L)$ with periodic boundary conditions $u(0)=u(L)$ and $u_{x}(0)=u_{x}(L)$.

Lemma 3.3. For periodic coefficients with minimal period $L>$ 0 , we have

$\operatorname{spec} \mathcal{L}=\bigcup_{v \in \mathrm{i}[0,2 \pi / L)} \operatorname{spec} \mathcal{L}_{\nu}$.

In particular, the following assertions are equivalent: (i) $\lambda \in \operatorname{spec} \mathcal{L}$;

(ii) $\left[D\left(\partial_{x}+v\right)^{2}+c\left(\partial_{x}+v\right)+a(x)-\lambda\right] u=0$ for some $u \in H_{\mathrm{per}}^{2}(0, L)$ and some $v \in \mathrm{i}[0,2 \pi / L)$;

(iii) $d(\lambda, v):=\operatorname{det}\left(\Phi_{\lambda}-\mathrm{e}^{v L}\right)=0$ for some $v \in \mathrm{i}[0,2 \pi / L)$.

The proof is a consequence of the Bloch-wave decomposition

$L^{2}(\mathbb{R}) \cong \bigoplus_{\nu \in \mathrm{i}[0,2 \pi / L)} L_{\mathrm{per}}^{2}(0, L)$

given by the isomorphism

$u(x)=\int_{v \in \mathrm{i}[0,2 \pi / L)} \mathrm{e}^{v x} w(x ; v) \mathrm{d} \nu$,

where $u \in L^{2}(\mathbb{R})$ and $w(x ; v)=w(x+L ; v)$ [16]. Since the direct computation of the Floquet exponents $v$ of the period map $\Phi_{\lambda}$ of the ODE (2.3) is often numerically unstable, condition (ii) is again preferable, from a numerical viewpoint, to the seemingly simpler condition (iii).

The operators $\mathcal{L}_{v}$ have compact resolvent for each $v$, and their spectra consist therefore of isolated eigenvalues with finite multiplicity whose real parts accumulate at $-\infty$. In particular, we denote the countably many eigenvalues of $\mathcal{L}_{0}$ by $\lambda_{j}$, which we order so that their real parts decrease as $j$ increases. The roots $\lambda_{j}$ of the complex analytic dispersion relation $d(\lambda, \nu)$ can again be continued globally in $v=\mathrm{i} \gamma$ with $\gamma \in \mathbb{R}$. In particular, it suffices to solve for $\gamma \in[0,2 \pi / L)$ since we necessarily have $\lambda_{j}(2 \pi \mathrm{i} / L)=\lambda_{\Pi(j)}(0)$ for some permutation $\Pi$ of $\mathbb{N}$.

Lemma 3.4. For periodic coefficients, the essential spectrum of $\mathcal{L}$ on $\mathbb{R}$ is an at most countable union of connected sets, each of which contains a point in the spectrum of $\mathcal{L}_{0}$, that is, an eigenvalue of the operator $\mathcal{L}$ considered on $(0, L)$ with periodic boundary conditions.

Note that the connected components do not need to contain a point at infinity. Isolas are possible, and the spectrum may not be connected on the Riemann sphere $\overline{\mathbb{C}}$ (see, for instance, Section 4.3 and Section 5.2).

Lastly, we briefly comment on the effect of coordinate transformations of the form $x \mapsto x-c_{*} t$ which correspond to changing the frame of reference in which spectra are computed. For constant coefficients, the passage to a comoving frame $\xi=x-c_{*} t$ simply introduces an additional drift term $c_{*} u \xi$ in the expression for $\mathcal{L}$. Thus, the eigenvalues $\Lambda$ in the frame $\xi$ can be computed from solutions $\lambda(v)$ of $d(\lambda, v)=0$ via $\Lambda=\lambda(v)-c_{*} \nu$. A similar result is true for periodic coefficients though the equation becomes time dependent, and we therefore have to consider the period map $\Psi_{T}$ of the linear PDE

$u_{t}=D u_{\xi \xi}+\left(c+c_{*}\right) u_{\xi}+a\left(\xi+c_{*} t\right) u$

with $T=L / c_{*}$.

Proposition 3.5 ([14]). The essential spectrum of $\Psi_{T}$ is of the form $\rho=\mathrm{e}^{\Lambda T}$ where $\Lambda=\lambda(v)-c_{*} \nu$, and $\lambda(v)$, with $v=\mathrm{i} \gamma$ and $\gamma \in[0,2 \pi / L)$, satisfies $d(\lambda(\nu), v)=0$. 
Thus, the computation of spectra in an arbitrary frame reduces to the solution of an eigenvalue problem of the type considered above. Note that spectral stability does not depend on the coordinate frame since the real part of the spectrum is independent of the chosen frame by Proposition 3.5.

\subsection{A priori estimates}

Since we assumed that all diffusion constants $d_{j}$ are positive, a straightforward scaling result for both $(\mathrm{C})$ and $(\mathrm{P})$ shows that for each fixed $\delta \in\left(0, \frac{\pi}{2}\right)$ there is a constant $R>0$ so that $\mathcal{L}$ does not have any spectrum with $|\lambda|>R$ and $|\arg \lambda|<\frac{\pi}{2}+\delta$.

It will turn out to be useful to consider the dispersion relations $d(\lambda, v)$ for purely imaginary temporal and spatial eigenvalues so that $\lambda=\mathrm{i} \omega$ and $\nu=\mathrm{i} \gamma$ for $\omega, \gamma \in \mathbb{R}$. We prove here that all real roots $(\omega, \gamma)$ of $d(\mathrm{i} \omega, \mathrm{i} \gamma)$ lie in bounded rectangles of $\mathbb{R}^{2}$ and provide estimates for these squares.

For constant coefficients, we assert (and refer to [9, Lemma 10] for the proof using Gershgorin circles) that any real solution $(\omega, \gamma)$ of $d(\mathrm{i} \omega, \mathrm{i} \gamma)=0$ satisfies

$(\omega, \gamma) \in\left[-|c| R_{0},|c| R_{0}\right] \times\left[-R_{0}, R_{0}\right]$

where

$R_{0}^{2}=\max _{1 \leq j \leq N} \frac{1}{d_{j}}\left(a_{j j}+\sum_{i=1, i \neq j}^{N}\left|a_{i j}\right|\right)$.

For periodic coefficients, we write $\mathcal{L}$ as the sum of the diagonal operator $\mathcal{L}^{0}$ with constant coefficients

$\mathcal{L}^{0}=\operatorname{diag}\left(d_{j}\right) \partial_{x x}+c \partial_{x}+\operatorname{diag}\left(\overline{a_{j j}}\right)$,

where $\bar{a}=\int_{0}^{L} a(x) \mathrm{d} x$, and the bounded remainder $\mathcal{L}^{1}$ which can be estimated in the operator norm on $L^{2}\left(\mathbb{R}, \mathbb{C}^{N}\right)$ by

$\left\|\mathcal{L}^{1}\right\| \leq \sup _{x \in[0, L]}\left|a(x)-\operatorname{diag}\left(\overline{a_{j j}}\right)\right|$,

where the norm on the right-hand side is the matrix norm induced by the norm used on $\mathbb{C}^{N}$ (the Euclidean norm on $\mathbb{C}^{N}$, for instance, induces the matrix norm $|A|=\sqrt{\sigma\left(A^{\mathrm{T}} A\right)}$ where $\sigma(B)$ denotes the spectral radius of the matrix $B)$. Using the explicit resolvent estimate

$$
\begin{gathered}
\left\|\left(\lambda-\mathcal{L}^{0}\right)^{-1}\right\| \leq \sup \left\{\left|-d_{j} k^{2}+c \mathrm{i} k+\overline{a_{j j}}-\lambda\right|^{-1} ;\right. \\
j=1, \ldots, N, k \in \mathbb{R}\},
\end{gathered}
$$

we see that the spectrum of $\mathcal{L}$ is contained in an $\left\|\mathcal{L}^{1}\right\|$ neighborhood of the spectrum

$\operatorname{spec} \mathcal{L}^{0}=\left\{-d_{j} k^{2}+c \mathrm{i} k+\overline{a_{j j}} ; j=1, \ldots, N, k \in \mathbb{R}\right\}$

of $\mathcal{L}^{0}$. Thus, any real root $(\omega, \gamma)$ of $d(\mathrm{i} \omega, \mathrm{i} \gamma)$ satisfies

$(\omega, \gamma) \in\left[-|c| R_{1},|c| R_{1}\right] \times\left[-R_{1}, R_{1}\right]$

where

$$
\begin{aligned}
R_{1}^{2} & =\max _{1 \leq j \leq N} \frac{1}{d_{j}}\left[\overline{a_{j j}}+\left\|\mathcal{L}^{1}\right\|\right] \\
& \leq \max _{1 \leq j \leq N} \frac{1}{d_{j}}\left[\overline{a_{j j}}+\sup _{x \in[0, L]}\left|a(x)-\operatorname{diag}\left(\overline{a_{k k}}\right)\right|\right] .
\end{aligned}
$$

A rough estimate for the real parts therefore is

$\operatorname{Re} \operatorname{spec}(\mathcal{L}) \leq 2\left(\max _{1 \leq j \leq N} \overline{a_{j j}}+\sup _{x \in[0, L]}\left|a(x)-\operatorname{diag}\left(\overline{a_{k k}}\right)\right|\right)$.

\subsection{Constant coefficients}

\subsubsection{Computing essential spectra using continuation}

For constant coefficients, we had seen that we can compute the essential spectrum of $\mathcal{L}$ by continuing the $N$ temporal eigenvalues $\lambda$ of the matrix $\mathcal{L}_{v}$ defined in (3.1) in the parameter $v=\mathrm{i} \gamma$. Thus, starting from $v=0$, say, where the temporal eigenvalues appear as eigenvalues of the matrix $a_{0}$, we can use the complex normalized eigenvalue equation

$\left[-D \gamma^{2}+c \mathrm{i} \gamma+a_{0}-\lambda\right] u=0, \quad\left\langle u_{\text {old }}, u\right\rangle=1$,

where $u_{\text {old }}$ denotes the eigenvector from a previous infinitesimal step in the continuation parameter $\gamma$ or the initially supplied solution at the beginning of the continuation. The condition

$\left\langle u_{\text {old }}, u\right\rangle=1$

is evaluated in the complex plane and therefore fixes the norm of the solution $u$ and its complex phase. Such a condition is necessary as nontrivial solutions $u$ to

$\left[-D \gamma^{2}+c \mathrm{i} \gamma+a_{0}-\lambda\right] u=0$

are, of course, not unique but come in group orbits $\left\{r \mathrm{e}^{\mathrm{i} \alpha} u ; \quad \alpha, r \in \mathbb{R}\right\}$. Eq. (3.4) can be replaced by any other condition that fixes a unique element in the group orbit of solutions. Bordering conditions similar to (3.4) will occur throughout this paper to enforce uniqueness of solutions.

\subsubsection{Testing stability}

Often, the spectrum is only computed to check whether a given homogeneous equilibrium is stable (i.e. whether its essential spectrum lies completely in the open left half-plane). For $N=2$, the spectrum is strictly stable if, and only if,

(i) $\operatorname{det}\left(a_{0}\right)>0$ and $\operatorname{tr}\left(a_{0}\right)<0$, and

(ii) $a_{22}^{0} d_{1}+a_{11}^{0} d_{2}<0$ or $\left(d_{1} a_{22}^{0}-d_{2} a_{11}^{0}\right)^{2}+4 d_{1} d_{2} a_{12}^{0} a_{21}^{0}<0$

where $a_{0}=\left(a_{i j}^{0}\right)$ (see e.g. [2]). For general $N$, connectedness of the essential spectrum on the Riemann sphere as stated in Lemma 3.2 immediately gives the following stability criterion.

Lemma 3.6. The essential spectrum of $\mathcal{L}$ is contained in the open left half-plane if, and only if, it does not intersect the imaginary axis.

Remark 3.7. When some of the diffusion coefficients $d_{j}$ vanish, then the equivalence stated in the lemma remains true provided $c \neq 0$ and all eigenvalues $\lambda_{j}$ of $a_{0}$ lie in the open left half-plane (curves with vertical asymptotes at some $\operatorname{Re}\left(\lambda_{j}\right)$ occur). 
To determine whether the essential spectrum intersects the imaginary axis, we do not need to calculate the entire essential spectrum. It suffices to compute the $2 N$ spatial eigenvalues $v$ for $\lambda \in i \mathbb{R}$ through continuation in $\lambda$. The above lemma then states that the equilibrium is stable provided $\operatorname{Re} v_{j} \neq 0$ for all $\lambda \in \mathrm{i} \mathbb{R}$ and each $j=1, \ldots, 2 N$. A strategy for determining stability therefore goes as follows:

(i) Compute the $2 N$ solutions $v_{j}(0)$ of $d(0, v)=0$ and find the associated nontrivial solutions $u_{j}$ of the equation

$$
\left[D v^{2}+c v+a_{0}\right] u=0, \quad|u|=1 .
$$

(ii) Follow each $\left(v_{j}, u_{j}\right)$ as solutions to

$$
\left[D v^{2}+c v+a_{0}-\mathrm{i} \omega\right] u=0, \quad\left\langle u_{\text {old }}, u\right\rangle=1
$$

by continuation in $\omega \in\left[0,|c| R_{0}\right]$ with $R_{0}$ as in Section 3.2, starting at $\omega=0$.

(iii) Stability is equivalent to $\operatorname{Re} v_{j}(\mathrm{i} \omega) \neq 0$ for all $\omega \in$ $\left[0,|c| R_{0}\right]$ and all $j$.

\subsubsection{Generic singularities}

When continuing roots $\lambda$ or $v$ of $d(\lambda, v)=0$ in the real parameters $v=\mathrm{i} \gamma$ or $\lambda=\mathrm{i} \omega$, it is of interest to know what the generic singularities are that one may encounter. On the level of the dispersion relation, this question can be easily answered. Continuation of $\lambda$ in $v=\mathrm{i} \gamma$ : We can always continue eigenvalues $\lambda$ as functions of $\nu=\mathrm{i} \gamma$ by the implicit function theorem unless $\partial_{\lambda} d(\lambda, v)=0$. Thus, suppose, without loss of generality, that $\partial_{\lambda} d(\lambda, v)=0$ at $\lambda=v=0$ so that

$d(\lambda, v)=\alpha_{20} \lambda^{2}+\alpha_{01} v+O\left(|v|^{2}+|\lambda \nu|+|\lambda|^{3}\right)$.

If $\alpha_{20} \alpha_{01} \neq 0$, then the Newton polygon shows that the solution set in $v=\mathrm{i} \gamma$ is given locally by the curves

$\lambda(\mathrm{i} \gamma)= \pm \sqrt{-\mathrm{i} \gamma \alpha_{01} / \alpha_{20}}+O(|\gamma|)$

for $\gamma \in \mathbb{R}$ close to zero. The coefficients $\alpha_{20}$ and $\alpha_{01}$ are real whenever the singularity occurs for real $\lambda$.

Due to analyticity, the equations $d=0$ and $\partial_{\lambda} d=0$ can be satisfied together either only at a discrete number of points $(\lambda, v)$ or else along curves. In the latter case, at least two branches of the essential spectrum coincide and, by Bézout's theorem, $d$ and $\partial_{\lambda} d$ have a common factor, which is, for instance, precluded if the diffusion rates are pairwise different [9, Lemma 10]. In the first case, the number of isolated double roots, counted with multiplicity, is equal to the degree of the resultant of $d(\cdot, v)$ and $\partial_{\lambda} d(\cdot, v)$ which is at most $2 N(2 N-1)$. In particular, for generic systems, we will not encounter any singularities during continuation in $v=\mathrm{i} \gamma$ since these should not occur for purely imaginary $v$. Thus, generically, we will be able to continue temporal eigenvalues in the real parameter $\gamma$ in a smooth fashion. A notable exception is the reversible situation $c=0$ where the dispersion relation depends analytically on $v^{2}=-\gamma^{2}$, so that temporal eigenvalues can collide on the real axis and split into complex conjugate pairs.

Continuation of $v$ in $\lambda=\mathrm{i} \omega$ : To determine stability, we proposed to continue the $2 N$ roots $v_{j}$ as functions of $\lambda=\mathrm{i} \omega$, whose singularities are of the form

$d(\lambda, \nu)=\alpha_{10} \lambda+\alpha_{02} \nu^{2}+O\left(|\nu|^{3}+|\lambda \nu|+|\lambda|^{2}\right)$.
If $d$ and $\partial_{\nu} d$ have no common factors, the number of double roots is again finite and, in fact, not larger than $N(2 N-1)$ by Lemma 4.5. The roots $v$ unfold in the same way as the roots $\lambda$ in (3.6) above. Since these singularities occur for discrete values of $\lambda$, they do typically not occur during continuation in $\lambda=\mathrm{i} \omega$.

\subsection{Periodic coefficients}

\subsubsection{Continuation-based computation of the essential spec- trum}

For periodic coefficients, we can compute the essential spectrum of $\mathcal{L}$ by continuing the countably many temporal eigenvalues $\lambda_{j}$ of the Bloch-wave operators $\mathcal{L}_{v}$ in the parameter $v=\mathrm{i} \gamma$. Supplementing the equation appearing in Lemma 3.3(ii) by an appropriate normalization condition, we obtain the complex boundary-value problem

$\left[D\left(\partial_{x}+\mathrm{i} \gamma\right)^{2}+c\left(\partial_{x}+\mathrm{i} \gamma\right)+a(x)-\lambda\right] u(x)=0$

$\int_{0}^{L}\left\langle u_{\text {old }}(x), u(x)\right\rangle \mathrm{d} x=1$,

where $u_{\text {old }}$ is the solution at a previous continuation step or the initially supplied solution at the beginning of the computation. Note that the integral condition is evaluated in the complex field $\mathbb{C}$ and therefore selects again an element in the real twodimensional group orbit.

If the linearization $\mathcal{L}$ arises from a translation invariant reaction-diffusion system as laid out in the introduction, then we typically need to solve the equation for the wave train and its temporal eigenvalues in tandem. Using the notation from Section 2 and normalizing the spatial period $L$ to unity, we therefore consider the boundary-value problem

$U_{x}=L F(U, c)$

$V_{x}=L\left[\partial_{U} F(U(x), c)+\lambda B-v\right] V$

$U(1)=U(0)$

$V(1)=V(0)$

$\int_{0}^{1}\left\langle U^{\prime}(x), U_{\text {old }}(x)-U(x)\right\rangle \mathrm{d} x=0$

$\int_{0}^{1}\left\langle V_{\text {old }}(x), V(x)\right\rangle \mathrm{d} x=1$,

corresponding to the travelling-wave ODE (2.6) and the eigenvalue problem (3.7), for $U \in \mathbb{R}^{N} \times \mathbb{R}^{N}$ and $V \in \mathbb{C}^{N} \times$ $\mathbb{C}^{N}$. Here, $\left(U_{\text {old }}, V_{\text {old }}\right)(x)$ denotes the solution at a previous continuation step or the initial solution at the beginning of the continuation, and we have added appropriate phase and normalization conditions to fix an element in the group orbit of solutions. Note that the complex normalization condition for $V$ is slightly different from the one used in (3.7) as it normalizes $\left(u, u_{x}\right)$ instead of only $u$. While theoretically equivalent, it turns out that one or the other may be more stable in numerical computations. We also remark that computations often run more reliably when the last equation in (3.8) is replaced by the nonlinear condition

$$
\int_{0}^{1}|V(x)|^{2} \mathrm{~d} x=1, \quad \int_{0}^{1} \operatorname{Im}\left\langle V_{\text {old }}(x), V(x)\right\rangle \mathrm{d} x=0 .
$$


We now focus on the case where $v=\mathrm{i} \gamma$ is purely imaginary as this gives the essential spectrum. We remark, however, that the considerations below remain true for $v \in \mathbb{C}$.

If we are given a solution $\left(U_{*}, V, \lambda, \mathrm{i} \gamma\right)$ of (3.8), then we can continue this solution numerically as a function of $v=\mathrm{i} \gamma$ by using a boundary-value solver such as AUTO. The generic singularities that we may encounter during continuation of (3.8) are identical to those for constant coefficients since both problems reduce to a single analytic equation in two complex variables; in particular, we do not expect that singularities arise during continuation in $\gamma$.

It remains to find initial solutions $(V, \lambda, \mathrm{i} \gamma)$ of the eigenvalue problem part of (3.8). Firstly note that, in the context of (3.8), $\lambda=0$ will always be an eigenvalue of $\mathcal{L}_{0}$ with eigenfunction $\partial_{x} u_{*}(x)$ due to translation invariance. Thus, $(U, V, \lambda, v)=\left(U_{*}, \partial_{x} U_{*}, 0,0\right)$ satisfies (3.8), and we can compute a curve $\lambda_{0}(v)$ of solutions to $d(\lambda, v)=0$ by continuation in $v=\mathrm{i} \gamma$ provided $\lambda=0$ is a simple eigenvalue of $\mathcal{L}_{0}$.

More generally, we may discretize the operator $\mathcal{L}_{0}$ with periodic boundary conditions using finite differences in space and solve the resulting matrix eigenvalue problem using packages such as LAPACK or MATLAB. Each of the resulting temporal eigenvalues $\lambda$ together with its eigenfunction $V$ can then be used as an initial guess for (3.8) at $\gamma=0$.

\subsubsection{Testing stability}

The following lemma gives conditions that guarantee spectral stability of spatially periodic equilibria.

Lemma 3.8. The essential spectrum of $\mathcal{L}$, with the exception of the eigenvalue $\lambda=0$, is contained in the open left half-plane provided the following conditions hold:

(i) The spectrum of $\mathcal{L}_{0}$ is contained in the open left half-plane except for the algebraically simple eigenvalue $\lambda=0$, and the curve $\lambda_{0}(\mathrm{i} \gamma)$ satisfies $\lambda_{0}^{\prime \prime}(0)>0$.

(ii) The origin $\lambda=0$ is not an eigenvalue of $\mathcal{L}_{v}$ for $v=\mathrm{i} \gamma \notin$ $2 \pi \mathrm{i} / L \mathbb{Z}$.

(iii) The spectrum of $\mathcal{L}$ does not intersect $\mathrm{i} \mathbb{R} \backslash\{0\}$.

To verify (i), we compute the spectrum of $\mathcal{L}_{0}$ and check that the eigenvalue $\lambda=0$ is simple and that there are no other eigenvalues in the closed right half-plane. Afterward, we continue $\lambda=0$ in $v=\mathrm{i} \gamma$ near $\gamma=0$ as outlined in the preceding section to see whether $\operatorname{Re} \lambda_{0}(\mathrm{i} \gamma)<0$ for $\gamma \neq 0$ near zero.

Condition (ii) is equivalent to the statement that the $2 N$ spatial Floquet exponents $v_{j}$ of the linear time- $L$ map $\Phi_{\lambda=0}$ that we defined in (2.5) are non-zero except for a single simple exponent $v_{1}=0$ that corresponds to the temporal eigenvalue $\lambda=0$. The exponents $v$ for $\lambda=0$ coincide with the Floquet exponents of the linearization

$U_{x}=L \partial_{U} F\left(U_{*}(x), c\right) U$

of the travelling-wave ODE about the wave train $U_{*}$, and condition (ii) is therefore equivalent to hyperbolicity of the wave train as a periodic orbit of (2.6). AUTO, for instance, has subroutines that compute these Floquet exponents together with the wave train $U_{*}$.

Condition (iii) can be checked as follows: Take the spatial Floquet exponents $v_{j}$ with $j=1, \ldots, 2 N$ of the wave train that were computed in the previous step at $\lambda=0$. For each of the $v_{j}$, we compute the corresponding Floquet eigenfunction $V$ by solving the linear boundary-value problem

$V_{x}=L\left[\partial_{U} F\left(U_{*}(x), c\right)-v\right] V+\epsilon H_{1}(x)$,

$V(1)=V(0), \quad \int_{0}^{1}\left\langle H_{2}(x), V(x)\right\rangle \mathrm{d} x=1$

for $(V, \epsilon)$ with $\epsilon \in \mathbb{C}$, where $H_{1}$ and $H_{2}$ are arbitrarily prescribed 1-periodic continuous functions. Note that (3.9) is linear in $(V, \epsilon)$, and it can been shown that it is uniquely solvable for any choice $\left(H_{1}, H_{2}\right)$ except when these lie in a certain subspace of $C^{0}\left([0,1], \mathbb{C}^{2 N}\right)$ of codimension one. Once we computed a Floquet eigenfunction $V_{j}$ for each $v_{j}$ at $\lambda=0$, we continue them in $\omega$ for $\omega \in\left(0,|c| R_{0}\right]$ with $R_{0}$ as in Section 3.2 as solutions $\left(U_{*}, V_{j}, \mathrm{i} \omega, v_{j}\right)$ of (3.8). Condition (iii) is met provided $\operatorname{Re} v_{j} \neq 0$ for all $\omega \in\left(0,|c| R_{0}\right]$ and all $j$.

\subsubsection{Group velocities, and Eckhaus instabilities}

Quantities relevant for the interaction and stability of spatiotemporally periodic travelling waves are their group velocity

$c_{\mathrm{g}}:=-\left.\frac{\mathrm{d} \lambda_{0}}{\mathrm{~d} \nu}\right|_{\nu=0} \in \mathbb{R}$,

which measures transport along the wave, and the coefficient

$\left.\frac{\mathrm{d}^{2} \lambda_{0}}{\mathrm{~d} \nu^{2}}\right|_{\nu=0} \in \mathbb{R}$

which determines whether the curve $\lambda_{0}(\mathrm{i} \gamma)$ extends into the left or the right half-plane near the origin. Continuation of these quantities in systems parameters allows us to detect sign changes of the group velocity and certain Eckhaus instabilities, in particular of sideband type, where the onset is via zero wavenumber [3].

To calculate the group velocity and the above coefficient, we consider the first-order system (2.3)

$U_{x}=L[A(x)+\lambda B] U, \quad U(1)=\mathrm{e}^{\mathrm{i} \gamma} U(0)$,

for $U \in \mathbb{C}^{2 N}$, with $L$ again rescaled to unity or, equivalently, the system

$V_{x}=L[A(x)+\lambda B-\mathrm{i} \gamma] V, \quad V(1)=V(0)$

where $U=\mathrm{e}^{\mathrm{i} \gamma x} V$. We set

$\lambda_{\mid}:=\left.\frac{\mathrm{d} \lambda_{0}}{\mathrm{~d} \nu}\right|_{\nu=0}, \quad \lambda_{\|}:=\left.\frac{\mathrm{d}^{2} \lambda_{0}}{\mathrm{~d} \nu^{2}}\right|_{\nu=0}$.

Differentiating (3.11) with respect to $v=\mathrm{i} \gamma$ and evaluating the resulting equations at $\gamma=0$, we obtain the system

$$
\begin{aligned}
& V_{\mid}^{\prime}=L\left[A(x) V_{\mid}+\left(\lambda_{\mid} B-1\right) V\right] \\
& V_{\|}^{\prime}=L\left[A(x) V_{\|}+2\left(\lambda_{\mid} B-1\right) V_{\mid}+\lambda_{\|} B V\right]
\end{aligned}
$$


on $(0,1)$ with periodic boundary conditions

$V_{\mid}(0)=V_{\mid}(1), \quad V_{\|}(0)=V_{\|}(1)$

for $V_{\mid}:=\partial_{\nu} V$ and $V_{\|}:=\partial_{\nu}^{2} V$ both in $\mathbb{R}^{2 N}$ and $\left(\lambda_{\mid}, \lambda_{\|}\right) \in \mathbb{R}^{2}$. Lastly, we add the integral conditions

$$
\int_{0}^{1}\left\langle V(x), V_{\mid}(x)\right\rangle \mathrm{d} x=0, \quad \int_{0}^{1}\left\langle V(x), V_{\|}(x)\right\rangle \mathrm{d} x=0,
$$

which ensure that both $V_{\mid}$and $V_{\|}$are $L^{2}$-orthogonal to the null space of the ODE for $V$. We mention that the scalar products in the integral conditions can, with $V=\left(V^{(1)}, V^{(2)}\right) \in \mathbb{R}^{2 N}$, be replaced by the PDE scalar products

$$
\begin{aligned}
& \int_{0}^{1}\left\langle V^{(1)}(x), V_{\mid}^{(1)}(x)\right\rangle \mathrm{d} x=0, \\
& \quad \int_{0}^{1}\left\langle V^{(1)}(x), V_{\|}^{(1)}(x)\right\rangle \mathrm{d} x=0,
\end{aligned}
$$

which are sometimes computationally more stable and reliable. The system (3.8) and (3.12)-(3.14) with $\lambda=v=0$ can now be solved uniquely for $(U, c),\left(V, V_{\mid}, V_{\|}\right)$and $\left(\lambda_{\mid}, \lambda_{\|}\right)$. Alternatively, using nontrivial solutions $V(x)$ and $W(x)$ of

$$
\begin{gathered}
V_{x}=L A(x) V, \quad V(0)=V(1), \quad W_{x}=-L A(x)^{t} W, \\
W(0)=W(1)
\end{gathered}
$$

and finding $\lambda_{\mid}$and $\tilde{V}(x)$ so that

$\tilde{V}_{x}=L\left[A(x) \tilde{V}+\left(\lambda_{\mid} B-1\right) V\right], \quad \tilde{V}(0)=\tilde{V}(1)$,

we have

$\lambda_{1}=\frac{\langle W, V\rangle_{L^{2}}}{\langle W, B V\rangle_{L^{2}}}, \quad \lambda_{\|}=\frac{\left\langle W, 2\left(\lambda_{\mid} B-1\right) \tilde{V}\right\rangle_{L^{2}}}{\langle W, B V\rangle_{L^{2}}}$.

\subsection{Implementation in AUTO}

We now discuss briefly how the strategies that we outlined above can be implemented in the continuation package AUTO and refer to the names of routines and constants as given in [4]; we write source code in FORTRAN syntax. Since AUTO uses only real arithmetics, dimension counting will always be done over the real numbers (unless explicitly stated otherwise).

\subsubsection{Periodic coefficients}

Implementing the system (3.8) in AUTO works as follows.

The constants file: Eq. (3.8) is a boundary-value problem, and we therefore set ips=4. The ODEs appearing in (3.8) involve $6 N$ real unknowns, namely $(U, V) \in \mathbb{R}^{2 N} \times \mathbb{C}^{2 N}$, and we therefore set ndim $=6 \mathrm{~N}$. We have $n b c=6 \mathrm{~N}$ real boundary conditions and nint $=3$ real integral conditions. Since the ODEs can be solved uniquely upon choosing initial conditions, we have effectively $6 N+3$ real equations and hence need the same number of variables plus one for continuation which gives $6 N+4$. In addition to the $6 N$ initial data for $(U, V)$, we have five real parameters at our disposal, namely $c, L, \gamma \in \mathbb{R}$ and $\lambda \in \mathbb{C}$. Thus, we may fix the period $L$ and use the four parameters $\gamma, c \in \mathbb{R}$ and $\lambda \in \mathbb{C}$ for continuation by setting $\mathrm{nicp}=4$ and specifying the four parameters in the array icp. It may be helpful for convergence to increase the number of Newton iterations itnw from its default value.

The equations file: The unknowns $(U, \operatorname{Re} V, \operatorname{Im} V) \in \mathbb{R}^{2 N} \times$ $\mathbb{R}^{2 N} \times \mathbb{R}^{2 N}$ are stored in $\mathrm{U}(1), \ldots, \mathrm{U}(6 \mathrm{~N})$, the period $L$ in $\operatorname{par}(11)$, and we use $\operatorname{par}(1), \ldots, \operatorname{par}(4)$ for $c, \gamma, \operatorname{Re} \lambda, \operatorname{Im} \lambda$. The periodic boundary conditions are defined in the subroutine bcnd via

$$
\begin{aligned}
& \text { do } j=1, \operatorname{ndim} \\
& \mathrm{fb}(j)=\mathrm{Uo}(j)-\mathrm{U} 1(j) \\
& \text { end do }
\end{aligned}
$$

The integral conditions are defined in icnd. We set

$$
\begin{aligned}
& f i(1)=0.0 \\
& \text { do } j=1, \operatorname{ndim} / 3 \\
& f i(1)=f i(1)+\operatorname{UPOLD}(j) *(\operatorname{UOLD}(j)-U(j)) \\
& \text { end do }
\end{aligned}
$$

for the phase condition of the wave train $U$, and use

$$
\begin{aligned}
& f i(2)=-1.0 \\
& f i(3)=0.0 \\
& \text { do } j=\text { ndim } / 3+1,2 * \text { ndim } / 3 \\
& \quad f i(2)=f i(2)+U(j) * U(j) \\
& \quad+U(j+\text { ndim } / 3) * U(j+\operatorname{ndim} / 3) \\
& \quad f i(3)=f i(3)+\operatorname{UOLD}(j) * U(j+\text { ndim } / 3) \\
& \quad-\operatorname{UOLD}(j+\text { ndim } / 3) * U(j) \\
& \text { end do. }
\end{aligned}
$$

for the normalization of the eigenfunction $V$.

Initial data: We assume that the period $L$, the wave train $U_{*}=$ $\left(u_{*}, u_{*}^{\prime}\right)$ and the associated wave speed $c$ are known. These can be obtained, for instance, from direct PDE simulations or from continuation in the travelling-wave ODE (2.6) beginning at a Hopf bifurcation point. We also assume that we found initial solutions for $\lambda, \gamma$ and the associated eigenfunction $V$ (see Section 3.4.1). This information needs to be stored in the subroutine stpnt or an external data file that can be read by AUTO (see [4]). It is recommended to scale the initial guess for the eigenfunction to have norm one so that it satisfies the integral condition; otherwise, convergence may be quite slow. Solving (3.9) and (3.12): We discuss now how Eq. (3.9) is solved to get the Floquet eigenfunction $V$ for a given Floquet exponent $v$. First, we pick functions $H_{1}$ and $H_{2}$ (for instance, constant functions). Note that (3.9) involves two real integral conditions and a real two-dimensional parameter $\epsilon$. We fix $v$ and continue instead in the real two-dimensional $\epsilon$ and the unused dummy parameter par (9): continuation in a dummy parameter in AUTO allows us to solve a linear or nonlinear system through Newton's method. Next, we continue to $\operatorname{Re} \epsilon=$ 0 using $\operatorname{Im} v$ as additional free parameter. Lastly, we continue to $\operatorname{Im} \epsilon=0$ using $v$ as free parameter.

Eq. (3.12) is solved analogously. It is affine in $\left(V_{\mid}, V_{\|}, \lambda_{\mid}, \lambda_{\|}\right)$ $\in \mathbb{R}^{2 N} \times \mathbb{R}^{2 N} \times \mathbb{R}^{2}$, and therefore almost any initial guess (for instance, constant functions) for $\left(V_{\mid}, V_{\|}, \lambda_{\mid}, \lambda_{\|}\right)$will give the correct solution by continuing in the dummy parameter 
$\operatorname{par}(9)$ and the active parameters $\left(\lambda, \lambda_{\mid}, \lambda_{\|}\right) \in \mathbb{C} \times \mathbb{R}^{2}$, starting at $\lambda=v=0$. We recommend to exclude $V_{\mid}, V_{\|}$from the pseudo-arclength computation by setting $n$ thu $=4 \mathrm{~N}$ followed by $4 N$ lines of the form 〈index of component) 0 .

\subsubsection{Constant coefficients}

The implementation for constant coefficients is similar to the one for periodic coefficients discussed above. While the eigenvalue problem (3.3) is only an algebraic equation, it is recommended to implement it as a boundary-value problem as in Section 3.5: this is done by setting ips $=4$ and choosing ntst=1 and ncol=2. In the following, we shall only comment on the differences to the implementation for periodic coefficients.

The constants file: Unless the equilibrium is to be continued in a parameter, we do not need to solve the nonlinear problem simultaneously. In this case, we have two integral conditions, three free parameters $(\gamma \in \mathbb{R}$ and $\lambda \in \mathbb{C})$, and the unknowns $u \in \mathbb{C}^{N}$ stored in $\mathrm{U}(1), \ldots, \mathrm{U}(2 \mathrm{~N})$ in AUTO. (It may also be convenient to use the files from the periodic case in the firstorder formulation with $U \in \mathbb{C}^{2 N}$.) We recommend disabling mesh adaptation by setting $i \mathrm{ad}=0$.

Initial data: Initial data for $\lambda, v$ and the eigenvector $u$ (or $U=$ $\left.\left(u, u_{x}\right)\right)$ can be imported from root-solving routines in packages such as MAPLE or MATHEMATICA applied to the dispersion relation $d(\lambda, v)=0$ for a fixed value of either $\lambda$ or $\nu$.

\section{Absolute spectra}

\subsection{Definition and characterization of the absolute spectrum}

As outlined in the introduction, the absolute spectrum arises naturally as follows: Take the linearization $\mathcal{L}$ about an asymptotically homogeneous or a periodic travelling wave and compute its spectrum on the interval $(-\ell, \ell)$ with fixed separated boundary condition at $x= \pm \ell$. The resulting spectra will depend on $\ell$ and on the boundary conditions. It is proved in [13] that these spectra converge, uniformly on compact subsets of $\mathbb{C}$ and in the symmetric Hausdorff distance, to a limiting spectral set as $\ell \rightarrow \infty$. The continuous (non-discrete) part of the limiting set is given by the absolute spectrum $\Sigma_{\text {abs }}$, defined below in Definition 1, which does not depend on the boundary conditions: As $\ell \rightarrow \infty$, each element of the absolute spectrum is approached by infinitely many eigenvalues of $\mathcal{L}$ which therefore cluster near the absolute spectrum. We emphasize that the results in [13], even though mostly formulated for constant coefficients, are valid for periodic coefficients.

Definition 1. For constant coefficients, we define the generalized absolute spectrum $\Sigma_{\text {abs }}^{m}$ with Morse index $m$ as the set of those $\lambda \in \mathbb{C}$ for which

$\operatorname{Re} v_{1} \geq \cdots \geq \operatorname{Re} v_{m}=\operatorname{Re} v_{m+1} \geq \cdots \geq \operatorname{Re} v_{2 N}$

where $v_{j}$ are the $2 N$ roots of $d(\lambda, v)$ repeated with multiplicity. The generalized absolute spectrum

$\Sigma_{\mathrm{abs}}^{*}:=\bigcup_{m=1}^{2 N-1} \Sigma_{\mathrm{abs}}^{m}$ is the union over all indices $m$, and the absolute spectrum is defined as

$\Sigma_{\mathrm{abs}}:=\Sigma_{\mathrm{abs}}^{N}$.

For periodic coefficients, we use the same definition with the eigenvalues $v$ replaced by the Floquet exponents of $\Phi_{\lambda}$.

Both notations $\Sigma_{\text {abs }}$ and $\Sigma_{\text {abs }}^{N}$ for the absolute spectrum will be used interchangeably. The generalized absolute spectrum with Morse index different from $N$ is usually meaningless for spectral properties of $\mathcal{L}$ from (2.1). It is, however, a natural first step towards the computation of the absolute spectrum. Note that each $\Sigma_{\text {abs }}^{m}$ is typically the union of curve segments that are glued together at singularities that we shall discuss in detail below. First, we note the absolute spectrum is also bounded to the right:

Remark 4.1. For both $(\mathrm{C})$ and $(\mathrm{P})$, a scaling result shows that for each fixed $\delta \in\left(0, \frac{\pi}{2}\right)$ there is a constant $R>0$ so that $\Sigma_{\text {abs }}^{N}$ does not contain any elements $\lambda$ with $|\lambda|>R$ and $|\arg \lambda|<\frac{\pi}{2}+\delta$.

The characterization of $\Sigma_{\text {abs }}^{*}$ in Definition 1 allows us to reformulate $\Sigma_{\text {abs }}^{*}$ using the system

$d\left(\lambda, v_{1}\right)=0, \quad d\left(\lambda, \nu_{2}\right)=0, \quad \nu_{2}-v_{1}=\mathrm{i} \gamma$

with $\gamma \in \mathbb{R}$. We see that $\lambda \in \Sigma_{\text {abs }}^{*}$ if either $\left(\lambda, v_{1}, v_{2}\right)$ are solutions of (4.1) for some non-zero real $\gamma$ or else if $\lambda$ and $\nu_{1}=\nu_{2}$ are solutions of (4.1) with $\partial_{\nu} d\left(\lambda, \nu_{1}\right)=0$. Setting $v=v_{1}$ and $v_{2}=v+\mathrm{i} \gamma$, we can remove the singularity of (4.1) at $\gamma=0$ by considering the system

$\mathcal{A}(\lambda, \nu ; \gamma)=\left(d(\lambda, \nu), \frac{d(\lambda, \nu)-d(\lambda, \nu+\mathrm{i} \gamma)}{\mathrm{i} \gamma}\right)=0$

so that $\mathcal{A}: \mathbb{C} \times \mathbb{C} \times \mathbb{R} \rightarrow \mathbb{C}^{2}$ and $\mathcal{A}(\lambda, v ; 0)=$ $\left(d(\lambda, v), d_{v}(\lambda, v)\right)$. Thus, $\lambda \in \Sigma_{\text {abs }}^{*}$ if, and only if, $(\lambda, v ; \gamma)$ satisfies (4.2). We shall call solutions $(\lambda, \nu)$ of $\mathcal{A}(\lambda, v ; 0)=0$ double roots.

In practice, it is often not feasible, or numerically not stable, to use the formulations (4.1) or (4.2) directly. Instead, it is typically more convenient to work with the original algebraic or differential system that leads to the solvability conditions (4.1) or (4.2), and we give those systems in Sections 4.3 and 4.2.4.

\subsection{Constant coefficients}

In Sections 4.2.1-4.2.3, we collect various useful properties of the absolute spectrum before commenting on the practical implementation of the continuation algorithm in Section 4.2.4.

\subsubsection{Continuation within the generalized absolute spectrum}

We first collect several properties of the system $\mathcal{A}(\lambda, \nu ; \gamma)=$ 0 . For each isolated solution $(\lambda, v)$ of $\mathcal{A}(\lambda, v ; \gamma)=0$ for some fixed $\gamma \geq 0$, we can define its multiplicity to be the (real) Brouwer degree $\operatorname{deg}(\mathcal{A}(\cdot, \cdot ; \gamma), 0)$ in the variable $(\lambda, \nu)$ evaluated at the solution $(\lambda, v)$. 
Lemma 4.2. The multiplicity is non-negative, and the multiplicity of an isolated double root $(\lambda, \nu)$ is one precisely when $\partial_{\lambda} d(\lambda, \nu) \neq 0$ and $\partial_{\nu v} d(\lambda, v) \neq 0$.

Proof. Since the derivative $\partial_{(\lambda, v)} \mathcal{A}$ is complex linear, it has a non-negative determinant when considered as a real $4 \times 4$ matrix which proves the first claim. The second statement follows since $\partial_{(\lambda, v)} \mathcal{A}$ is, in this case, block upper triangular with diagonal entries given as non-zero complex multiples of the identity, such that $\operatorname{det} \partial_{(\lambda, v)} \mathcal{A}>0$.

We say that an isolated double root is simple if it has multiplicity one. If a solution is not isolated, we say it has multiplicity $\infty$.

In the following, we consider various homotopies by allowing the coefficients $D, c, a_{0}$ and $\gamma$ to depend on a homotopy parameter $\tau \in[0,1]$. The resulting functions will be denoted by $\mathcal{A}_{\tau}(\lambda, \nu)$, omitting the dependence on $\gamma=\gamma(\tau)$. The homotopy invariance of the Brouwer degree gives the following result.

Lemma 4.3. The number of solutions $(\lambda, \nu)$ of $\mathcal{A}_{\tau}(\lambda, \nu)=0$ inside a ball $G \subset \mathbb{C}^{2}$, counted with multiplicity, is independent of $\tau$ provided there are no roots on the boundary $\partial G$ for each $\tau \in[0,1]$.

Next, we prove that the assumption in the preceding lemma is automatically met provided the ball $G$ has sufficiently large diameter.

Lemma 4.4. If the diffusion coefficients are pairwise distinct so that $d_{i} \neq d_{j}$ for $i \neq j$, then there exists a number $R>0$, depending only on $|D|,\left|a_{0}\right|,|c|$ and $|\gamma|$, such that every solution $\left(\lambda, \nu_{1}, v_{2}\right)$ of (4.1) satisfies

$|\lambda|+\left|v_{1}\right|+\left|\nu_{2}\right| \leq R$.

Proof. Suppose that the claim is wrong so that, for a certain fixed $\gamma$, there are solutions $(\lambda, \nu)$ of (4.1) for which $|\lambda|,\left|\nu_{1}\right|$, or $\left|\nu_{2}\right|$ are arbitrarily large. In this case, a straightforward estimate of the equation $d(\lambda, v)=0$ shows that $|v| \equiv \pm \sqrt{\lambda / d_{i}}$ for some $i$ as $|\lambda|$ or $|\nu|$ tend to infinity. Since $d_{i} \neq d_{j}$ for $i \neq j$, this implies $\left|\operatorname{Im}\left(v_{1}-v_{2}\right)\right| \rightarrow \infty$ whenever $\operatorname{Re} v_{1}=\operatorname{Re} v_{2}$, and therefore $|\gamma| \rightarrow \infty$ as well. This contradicts the assumption that $\gamma$ is fixed and therefore shows that $\left(\lambda, v_{1}, \nu_{2}\right)$ stays in a bounded region.

Lemma 4.5. Assume that $d_{i} \neq d_{j}$ for $i \neq j$, then there are precisely $N(2 N-1)$ double roots, i.e. solutions to $\mathcal{A}(\lambda, v ; 0)=$ 0 , when counted with multiplicity.

Proof. We choose a homotopy of $\mathcal{A}(\lambda, v ; 0)=0$ to the equation with $c=0$ and $a=\operatorname{diag}\left(a_{j}\right)$. On account of Lemmas 4.3 and 4.4 , the number of roots of $\mathcal{A}_{\tau}(\lambda, v ; 0)$ does not change during the homotopy. The resulting diagonal equation has the $N$ double roots $(\lambda, v)=\left(a_{j}, 0\right)$ which are easily seen to have multiplicity one. The remaining double roots are solutions to

$d_{i} v^{2}+a_{i}-\lambda=0, \quad d_{j} v^{2}+a_{j}-\lambda=0$, that is, to

$\lambda=d_{i} v^{2}+a_{i}, \quad v^{2}=-\frac{a_{i}-a_{j}}{d_{i}-d_{j}}$

for a given pair $(i, j)$ with $1 \leq i<j \leq N$. Choosing the $a_{j}$ appropriately, the above system has $N(N-1)$ distinct solutions. We claim that each solution has Brouwer degree equal to 2 . Indeed, differentiating the dispersion relation

$d(\lambda, \nu)=\prod_{j=1}^{N}\left[\lambda-d_{j} v^{2}-a_{j}\right]$

and computing the Taylor jet at the solutions $\left(\lambda_{*}, \nu_{*}\right)$, we obtain

$d(\lambda, \nu)=a\left(\lambda-\lambda_{*}\right)^{2}-b\left(v-v_{*}\right)^{2}+O(3)$

with $a \neq 0$. In particular, $d(\lambda, \nu)+\varepsilon$ has two simple double roots at $\lambda=\lambda_{*} \pm \sqrt{\varepsilon / a}+O(\epsilon)$ and $v=v_{*}$. The additivity and homotopy invariance of the degree shows that the multiplicity is two. Altogether, we have found $N+2 N(N-1)=N(2 N-1)$ roots which proves the lemma.

We can now show that the generalized absolute spectrum consists of at most $N(2 N-1)$ curves that are parameterized by $\gamma$ :

Proposition 4.6. The generalized absolute spectrum is given by $\Sigma_{\text {abs }}^{*}=\bigcup_{\gamma \geq 0}\left\{\lambda_{j}(\gamma) ; j=1, \ldots, N(2 N-1)\right\}$,

where $\lambda_{j}(\gamma)$ denotes the $\lambda$-component of the solutions to $\mathcal{A}(\lambda, v ; \gamma)=0$. Moreover, $\lambda_{j}(\gamma)$ can be chosen to be continuous in $\gamma$. In particular, $\Sigma_{\text {abs }}^{*}$ consists of at most $N(2 N-$ 1) connected components, each containing a double root and the point at infinity (when considered on $\overline{\mathbb{C}}$ ).

Proof. The representation of $\Sigma_{\text {abs }}^{*}$ follows from the previous discussion and the homotopy invariance of the degree in a ball of radius $R$ near each fixed finite $\gamma$, and therefore for all $\gamma$. To prove continuity, we have to show that the set $\mathcal{C}:=$ $\{(\lambda, v, \gamma) ; \mathcal{A}(\lambda, v ; \gamma)=0\}$ is the union of $N(2 N-1)$ continuous curves $\left(\lambda_{j}, v_{j}\right)(\gamma)$. It suffices to prove this property locally, and we therefore work in a fixed box $\mathcal{B}=\mathcal{B}_{(\lambda, v)} \times \mathcal{B}_{\gamma} \subset$ $\mathbb{C}^{2} \times \mathbb{R}^{+}$near a given root $\left(\lambda_{*}, v_{*}, \gamma_{*}\right)$ of $\mathcal{A}$ with multiplicity $r$. By Sard's theorem, there exists a sequence $c_{k} \in \mathbb{C}^{2}$ with $c_{k} \rightarrow 0$ as $k \rightarrow \infty$ so that each $c_{k}$ is a regular value of $\mathcal{A}: \mathbb{C}^{2} \times \mathbb{R}^{+} \rightarrow \mathbb{C}^{2}$. In particular, the preimages $\mathcal{A}^{-1}\left(c_{k}\right) \cap \mathcal{B}$ are smooth one-dimensional submanifolds, and therefore the disjoint union of one-dimensional embedded curves. We claim that each of these curves can be parameterized by $\gamma$ : Indeed, if this were not possible, we would readily obtain a contradiction to the fact that there are precisely $r$ roots of $\mathcal{A}(\cdot, \cdot ; \gamma)$ in $\mathcal{B}_{(\lambda, v)}$ for each fixed $\gamma \in \mathcal{B}_{\gamma}$ by degree arguments. Thus, for each $k$, we can parameterize the roots of $\mathcal{A}$ inside $\mathcal{B}$ by $r$ disjoint continuous curves $\left(\lambda_{j}^{(k)}, v_{j}^{(k)}\right)(\gamma)$, and taking the limit $k \rightarrow \infty$ gives the desired characterization of $\Sigma_{\text {abs }}^{*}$.

To summarize, to compute the generalized absolute spectrum, it suffices to calculate all double roots and to 
subsequently continue the curves of $\Sigma_{\mathrm{abs}}^{*}$ that emanate from the double roots in $\gamma$ from $\gamma=0$ to $\gamma=\infty$.

We remark that double roots may coalesce in certain situations:

Lemma 4.7. If $a$ is triangular and $N \geq 2$, then there are at least two degenerate double roots $\left(\lambda_{*}, v_{*}\right)$ of the dispersion relation which are double roots with respect to both $\nu$ and $\lambda$ so that

$d\left(\lambda_{*}+\lambda, v_{*}+v\right)=\alpha_{20} \lambda^{2}+\alpha_{02} v^{2}+O\left(|\lambda|^{3}+|v|^{3}\right)$.

Proof. It suffices to consider $N=2, D=(1, \delta)$ with $\delta \neq 0$,

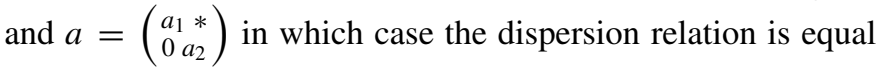
to $d(\lambda, v)=d_{1}(\lambda, v) d_{2}(\lambda, v)=0$ with $d_{1}(\lambda, v)=v^{2}+c v-$ $\lambda+a_{1}$ and $d_{2}(\lambda, v)=\delta v^{2}+c v-\lambda+a_{2}$. In particular, there are $\left(\begin{array}{l}4 \\ 2\end{array}\right)=6$ double roots for $\delta \neq 1$ which satisfy $d=0$ and $\partial_{\nu} d=\left(\partial_{\nu} d_{1}\right) d_{2}+d_{1} \partial_{\nu} d_{2}=0$.

The solutions to $d_{1}=0$ and $d_{2}=0$ are given by $\lambda_{1}=$ $v^{2}+c v+a_{1}$ and $\lambda_{2}=\delta v^{2}+c v+a_{2}$, respectively, where $v$ is arbitrary. These give rise to spatial double roots provided $d_{2}\left(\lambda_{1}, v\right)=0$ or $d_{1}\left(\lambda_{2}, v\right)=0$, respectively, that is, when $\delta v^{2}+c v-\left(v^{2}+c v+a_{1}\right)+a_{2}=0$ or $v^{2}+c v-\left(\delta v^{2}+\right.$ $\left.c v+a_{2}\right)+a_{1}=0$. This is the case for $v_{j}^{ \pm}= \pm \sqrt{(-1)^{j} \frac{a_{2}-a_{1}}{1-\delta}}$. The remaining two of the six double roots are the roots $v_{1}=$ $-\frac{c}{2}$ and $v_{2}=-\frac{c}{2 \delta}$ of the dispersion relations $d_{1}$ and $d_{2}$, respectively.

If some of the diffusion coefficients are equal $\left(d_{i}=d_{j}\right.$ for appropriate indices $i \neq j$ ), we cannot a priori exclude that branch points 'disappear' at infinity. In fact, in the explicit decoupled model problem that we utilized in the proof of Lemma 4.5, a double branch point 'crosses' the point at infinity when $d_{i}-d_{j}$ crosses zero.

\subsubsection{Testing absolute stability}

We shall show that, for constant-coefficient operators, the absolute spectrum $\Sigma_{\text {abs }}^{N}$ is connected in $\overline{\mathbb{C}}$. Since Remark 4.1 shows furthermore that it lies in an acute sector that opens up along the negative real axis, it suffices to check whether the absolute spectrum has a non-zero intersection with the imaginary axis to establish stability or instability.

Lemma 4.8. The absolute spectrum $\Sigma_{\mathrm{abs}}^{N}$ is connected in $\overline{\mathbb{C}}$ and contains the point at infinity. Furthermore, the absolute spectrum $\Sigma_{\mathrm{abs}}^{N}$ is contained in the open left half-plane if, and only if, it does not intersect the imaginary axis.

Proof. We argue by contradiction. Thus, suppose that $\tilde{\Sigma}$ is a non-empty, compact subset of $\Sigma_{\text {abs }}^{N}$ so that there is a smooth Jordan curve $\Gamma$ in $\mathbb{C}$ with $\Gamma \cap \Sigma_{\text {abs }}^{N}=\emptyset$ and int $\Gamma \cap \Sigma_{\text {abs }}^{N}=\tilde{\Sigma}$. The idea is to show that the spectrum of $\mathcal{L}$ on $(-\ell, \ell)$ with appropriate boundary conditions cannot accumulate on $\tilde{\Sigma}$ as $\ell \rightarrow \infty$ in contradiction to [13, Theorem 5]. Since $\Gamma$ does not intersect the absolute spectrum with Morse index $N$, the eigenvalues $v_{j}$ of $A+\lambda B$ satisfy

$\operatorname{Re} v_{1} \geq \cdots \geq \operatorname{Re} v_{N}>\operatorname{Re} v_{N+1} \geq \cdots \geq \operatorname{Re} v_{2 N}$ for all $\lambda \in \Gamma$. We denote the $N$-dimensional generalized eigenspaces associated with the $N$ smallest and largest eigenvalues of $A+\lambda B$ in the above ordering by $E^{S}(\lambda)$ and $E^{u}(\lambda)$, respectively: These spaces are well defined, unique and analytic in $\lambda$ for $\lambda$ in a neighbourhood $\mathcal{U}$ of $\Gamma$. Next, pick $\lambda_{0} \in \Gamma$ and an $N$-dimensional subspace $E^{\mathrm{bc}}$ with

$E^{\mathrm{bc}} \oplus E^{u}(\lambda)=\mathbb{C}^{2 N}, \quad E^{\mathrm{bc}} \oplus E^{s}(\lambda)=\mathbb{C}^{2 N}$

for $\lambda=\lambda_{0}$. Analyticity then implies that (4.3) is true for all $\lambda \in \mathcal{U}$ except possibly for finitely many $\lambda$. Redefining $\Gamma$ if necessary, we can therefore assume that (4.3) is true for all $\lambda \in \Gamma$.

We set our boundary conditions by choosing a matrix $Q_{-}^{\text {bc }}=$ $Q_{+}^{\text {bc }}$ with null space equal to $E^{\text {bc }}$. Eq. (4.3) shows that [13, Hypothesis 7] is met, and [13, Proposition 5] now asserts that there are numbers $M \geq 0$ and $\ell_{*} \gg 1$ such that the spectrum of $\mathcal{L}$ on $(-\ell, \ell)$ with the boundary conditions (1.3) cannot intersect $\Gamma$ for $\ell \geq \ell_{*}$ and contains precisely $M$ elements in the interior of $\Gamma$ for $\ell=\ell_{*}$. Thus, the number of eigenvalues in the interior of $\Gamma$ is equal to $M$ for all $\ell \geq \ell_{*}$. We emphasize that (4.3), and therefore the above statement, remains true if we change $E^{\mathrm{bc}}, A$, and $B$ slightly.

Next, pick an element $\lambda_{1}$ in the non-empty set $\tilde{\Sigma}$. Upon transforming the matrix $A+\lambda_{1} B$ into Jordan normal form, it is easy to see that there are matrices $C_{0}$ and $C_{1}$ of arbitrarily small norm so that the eigenvalues $v_{j}$ of

$A+\lambda_{1} B+C_{0}+\left(\lambda-\lambda_{1}\right) C_{1}$

satisfy

$\operatorname{Re} v_{1} \geq \cdots \geq \operatorname{Re} v_{N-1}>\operatorname{Re} v_{N}=\operatorname{Re} v_{N+1}$

$>\operatorname{Re} v_{N+2} \geq \cdots \geq \operatorname{Re} v_{2 N}$,

$\operatorname{Im} v_{N} \neq \operatorname{Im} v_{N+1}$

at $\lambda=\lambda_{1}$ and

$\left.\frac{\mathrm{d} \operatorname{Im}\left(v_{N}-v_{N+1}\right)}{\mathrm{d} \lambda}\right|_{\lambda=\lambda_{1}} \neq 0$.

In particular, we may choose $C_{0}$ and $C_{1}$ so small that the statements in the previous paragraph are also true for (4.4). On the other hand, (4.5) and (4.6) show that [13, Hypothesis 8] is satisfied near $\lambda=\lambda_{1}$, and [13, Theorem 5] now implies that the number of eigenvalues of $\mathcal{L}$, posed on $(-\ell, \ell)$ with the boundary conditions (1.3), in a small disk centered at $\lambda_{1}$ becomes unbounded as $\ell \rightarrow \infty$. This contradicts the statement established before that this number is equal to $M$ which is independent of $\ell$. The second statement of the lemma follows from Remark 4.1.

\subsubsection{Generic singularities}

The generalized absolute spectrum $\Sigma_{\text {abs }}^{*}$ does typically not have any singularities except that curves may begin or end at double roots: For a double root at the origin, we have $\lambda=\alpha \nu^{2}$ which gives $v_{1}=-v_{2}= \pm \mathrm{i} \gamma / 2$ for $\gamma \geq 0$ along the curve $\lambda=-\gamma^{2} \alpha / 4$ of generalized absolute spectrum. However, even though we may continue curves smoothly in $\Sigma_{\text {abs }}^{*}$, the Morse index $m$ may jump along these curves. This occurs typically 


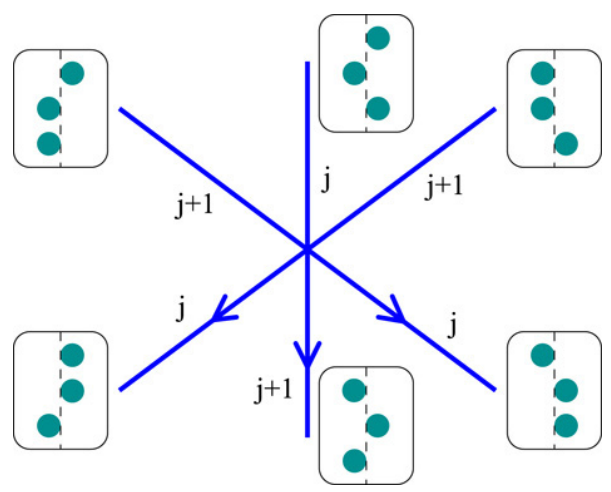

Fig. 1. A triple-point singularity of the generalized absolute spectrum in the complex $\lambda$-plane. The curves are oriented by increasing $\gamma$, the numbers along the curves indicate the Morse index, and the insets show the relevant spatial eigenvalues in the complex plane where we plot $\operatorname{Re}\left(v\left(\lambda_{\text {triple }}\right)\right)+i \mathbb{R}$ as a dashed line.

at triple points where $\operatorname{Re} v_{j+1}=\operatorname{Re} v_{j+2}=\operatorname{Re} v_{j+3}$ and $\operatorname{Im} v_{j+1}>\operatorname{Im} v_{j+2}>\operatorname{Im} v_{j+3}$. Triple points should form a discrete subset of the generalized absolute spectrum. Typically, $\lambda=\lambda_{\text {triple }}+b_{l}\left(v-v_{l}\right)+O\left(\left|v-v_{l}\right|^{2}\right)$ for $l=j, j+1, j+2$ near these singularities, and the resulting bifurcation picture is readily computed under the assumption that the coefficients $b_{l}$ are different from each other (see Fig. 1).

The Morse index drops from $j+1$ to $j$ along two of the curves as they cross the singularity. Between these two curves, there is a curve of generalized absolute spectrum which also crosses the singularity and along which the Morse index increases. The Morse index increase occurs along the curve where $\operatorname{Re} v_{1}=\operatorname{Re} v_{3}$, i.e. where the difference of imaginary parts is given by the sum of the two other differences of imaginary parts.

Note that if we insist on following curves with constant Morse index (rather than preserving smoothness during the continuation), then we lose a curve with Morse index $j+1$ and create a curve with Morse index $j$. Furthermore, if we follow curves with Morse index $j$, the parameter $\gamma$ jumps to a lower value as we cross the singularity: In particular, we cannot enforce by local considerations only that curves of constant Morse index continue to $\gamma=\infty$, although we may well be able to continue them in $\lambda$. We emphasize that the absolute spectrum is nevertheless connected in $\overline{\mathbb{C}}$, see Lemma 4.8.

The singularity $\mathrm{d} \nu_{1} / \mathrm{d} \lambda=\mathrm{d} \nu_{2} / \mathrm{d} \lambda$ at $v_{j}^{*}$ with $j=1,2$ in the generalized absolute spectrum typically requires an additional systems parameter, but can be observed for real $\lambda$ without external parameter. To leading order, we find from the dispersion relation that $v_{j}=v_{j}^{*}+a \lambda+b_{j} \lambda^{2}$ for $j=1,2$ and therefore

$\operatorname{Re}\left(v_{1}-v_{2}\right)=\operatorname{Re}\left[\left(b_{1}-b_{2}\right) \lambda^{2}\right]=0$.

The solutions of $\operatorname{Re}\left[\left(b_{1}-b_{2}\right) \lambda^{2}\right]=0$ form a cross (in $\mathrm{x}$-shape if $b_{1}-b_{2}$ is real), and $\gamma$ increases towards the singularity on one of the curves and decreases along the other curve towards the singularity. The Morse indices are the same on all four curves. This singularity occurs, for instance, on a real bounded interval of $\Sigma_{\text {abs }}^{*} \cap \mathbb{R}$ whose endpoints are double roots.
Similarly, we can encounter two disjoint pairs of spatial eigenvalues with the same real part, in a robust fashion for real $\lambda=\lambda_{*}$, or with an additional parameter for $\lambda$ complex. We expect $6=\left(\begin{array}{l}4 \\ 2\end{array}\right)$ smooth curves of generalized absolute spectrum to intersect at such a value of $\lambda$. If $\lambda$ is real, then two of these curves coincide and lie on the real axis due to the additional symmetry of spatial eigenvalues with respect to complex conjugation.

\subsubsection{The algorithm in practice}

Computationally, it is more reliable and convenient to replace the dispersion relation $d(\lambda, \nu)$ by the original algebraic equation. Using the definition

$\mathcal{D}(\lambda, v):=D v^{2}+c v+a_{0}-\lambda$,

the system $\mathcal{A}(\lambda, v ; \gamma)=0$ is equivalent to solving

$\mathcal{D}(\lambda, v) u=0$,

$[D(2 v+\mathrm{i} \gamma)+c](u+\mathrm{i} \gamma v)+\mathcal{D}(\lambda, v) v=0$,

together with the normalization

$\left\langle u_{\text {old }}, u\right\rangle=1, \quad\left\langle v_{\text {old }}, u\right\rangle-\left\langle u_{\text {old }}, v\right\rangle-\mathrm{i} \gamma\left\langle v_{\text {old }}, v\right\rangle=0$

for $(\lambda, v, \gamma, u, v)$, where $\left(u_{\text {old }}, v_{\text {old }}\right)$ are the solutions taken at a previous continuation step.

Proof. For $\gamma \neq 0$, we start with (4.1) and see that it is equivalent to the system

$\mathcal{D}(\lambda, v) u=0, \quad\langle u, u\rangle=1$

$\mathcal{D}(\lambda, v+\mathrm{i} \gamma) w=0, \quad\langle w, w\rangle=1$

with the additional unknowns $(u, w)$. If we assume that $u_{\text {old }}$ and $w_{\text {old }}$ are solutions to this system, or close to solutions, then we may replace the above system by

$\mathcal{D}(\lambda, v) u=0, \quad\left\langle u_{\text {old }}, u\right\rangle=1$

$\mathcal{D}(\lambda, v+\mathrm{i} \gamma) w=0, \quad\left\langle w_{\text {old }}, w\right\rangle=1$.

We write $w$ in the form $w=u+\mathrm{i} \gamma v$ so that the second equation becomes

$0=\mathcal{D}(\lambda, v+\mathrm{i} \gamma)(u+\mathrm{i} \gamma v)=[\mathcal{D}(\lambda, v)-\mathcal{D}(\lambda, v+\mathrm{i} \gamma)] u$

$$
+\mathcal{D}(\lambda, v+\mathrm{i} \gamma) \mathrm{i} \gamma v
$$

where we used the equation for $u$. Dividing by $\mathrm{i} \gamma$ and using (4.7), we get

$[D(2 v+\mathrm{i} \gamma)+c] u+\mathcal{D}(\lambda, v+\mathrm{i} \gamma) v=0$.

The normalization condition $\left\langle w_{\text {old }}, w\right\rangle=1$ for $w$ becomes

$$
\begin{aligned}
1 & =\left\langle u_{\text {old }}+\mathrm{i} \gamma v_{\text {old }}, u+\mathrm{i} \gamma v\right\rangle \\
& =1+\mathrm{i} \gamma\left\langle u_{\text {old }}, v\right\rangle+\mathrm{i} \gamma\left\langle v_{\text {old }}, u+\mathrm{i} \gamma v\right\rangle
\end{aligned}
$$

and therefore

$\left\langle u_{\text {old }}, v\right\rangle+\left\langle v_{\text {old }}, u+\mathrm{i} \gamma v\right\rangle=0$.

The computations for $\gamma=0$ are similar and we therefore omit them. 


\subsection{Periodic coefficients}

The initial set-up for periodic coefficients is similar. The dispersion relation is now given by

$d(\lambda, v)=\operatorname{det}\left[\Phi_{\lambda}-\mathrm{e}^{\nu L}\right]$,

and we shall use the regularized system

$\mathcal{D}(\lambda, v) u=0$,

$\left[D\left(2\left(\partial_{x}+v\right)+\mathrm{i} \gamma\right)+c\right](u+\mathrm{i} \gamma v)+\mathcal{D}(\lambda, v) v=0$,

where

$\mathcal{D}(\lambda, v)=D\left(\partial_{x}+v\right)^{2}+c\left(\partial_{x}+v\right)+a(x)-\lambda$,

together with the normalization conditions

$\int_{0}^{L}\left\langle u_{\text {old }}, u\right\rangle \mathrm{d} x=1$

$\int_{0}^{L}\left[\left\langle v_{\text {old }}, u\right\rangle-\left\langle u_{\text {old }}, v\right\rangle-\mathrm{i} \gamma\left\langle v_{\text {old }}, v\right\rangle\right] \mathrm{d} x=0$

for $u$ and $v$. It suffices here to consider $\gamma \in[0, \pi / L)$.

Regarding instability of the absolute spectrum, we can conclude that the existence of an unstable isola of essential spectrum implies unstable absolute spectrum provided it lies on the boundary of the component of the resolvent set where the Morse index of the period map $\Phi_{\lambda}$ is $N$. This observation is a consequence of the more general fact that isolas of essential spectrum contain absolute spectrum of a certain Morse index (see [9, Theorem 3.3] and [10] for details).

Continuing a curve of generalized absolute spectrum starting at double roots proceeds as for constant coefficients. However, neither the generalized absolute spectrum nor the absolute spectrum of a given index are, in general, connected, and the initial computation of double roots becomes therefore more important. For periodic coefficients, there exist infinitely many double roots for $\gamma=0$ even though each bounded region of the complex plane typically contains only finitely many. In addition, it is more complicated to find double roots in the first place: in fact, we do not know of any systematic way of locating double roots in a given region of the complex plane.

Instead of starting continuation at a double root, one may, of course, start at any given element of the (generalized) absolute spectrum, and we therefore discuss now possible strategies for locating elements of the (generalized) absolute spectrum. These strategies involve continuing solutions of (3.8), where $v=\eta+\mathrm{i} \gamma$, in either $(\gamma, \lambda, L)$ or $(\eta, \lambda, L)$ or $(\nu, \operatorname{Re} \lambda, L)$. Continuation in $(\gamma, \lambda, L)$ for fixed and possibly non-zero $\eta$ corresponds to computing the essential spectrum of $\mathcal{L}$ in an exponentially weighted space with exponential rate $\eta$.

Firstly, consider an intersection point of two curve segments of essential spectrum, possibly for $\eta \neq 0$. Unless this point is a root of $\partial_{\lambda} d(\lambda, v)$, it lies in the generalized absolute spectrum, because two Floquet exponents have the same real part, and it can be used as a starting point for continuation. In fact, a Jordan curve of essential spectrum that does not contain further essential spectrum continues in $\eta$ either to a self intersecting curve or to a double root [10].
Secondly, we discuss the case when (generalized) absolute spectrum intersects the real axis. If $\lambda$ lies in the generalized absolute spectrum, then the two associated Floquet multipliers have the same modulus. If $\lambda$ is, in fact, real, then the two Floquet multipliers are either non-real complex conjugates, or they form a double root, or they are real and of opposite sign:

If the Floquet multipliers are non-real, simple and complex conjugates, then so are the associated Floquet exponents $v$. A small change in the real part of $\lambda$ or in $\eta=\operatorname{Re} v$ does not change the property that the exponents are complex conjugates and, consequently, the generalized absolute spectrum intersects the real axis in an interval with open interior. The end points of these intervals are double roots. Thus, continuation of $v=\eta+$ i $\gamma$ in $\lambda \in \mathbb{R}$ may lead to double roots in the generalized absolute spectrum. By symmetry of Floquet exponents, the computation of real intervals using (3.8) requires the continuation of only one Floquet exponent.

If there are two real simple Floquet multipliers of opposite sign for real $\lambda$, then these multipliers will stay real if $\lambda$ is varied on the real axis. Thus, on account of the Cauchy-Riemann equations, changing the imaginary part of $\lambda$ will, to leading order, only change the imaginary part of the Floquet multipliers, which shows that the corresponding curve of generalized absolute spectrum intersects the real axis transversely with a vertical tangent. Hence, continuing for real $\lambda$ a pair of Floquet multipliers with opposite sign in $v$ may lead to the location of such a crossing point.

Other typical singularities of the absolute spectrum on the real axis are as described in Section 4.2.3.

We refer to Section 5 (see also [9, Chapter 4.4] and [10]) for examples where we use exponential weights to locate absolute spectrum.

\subsection{Continuation, and implementation in AUTO}

In the case of constant coefficients, we first calculate all double roots $(\lambda, v)$, i.e. all roots of $\mathcal{A}(\lambda, v ; 0)=0$, and subsequently nontrivial solutions $u$ and $v$ of the linear equation (4.8). Starting from each of these at most $N(2 N-1)$ points, we then continue solutions of (4.8) and (4.9) in $\gamma$.

Afterward, we reconstruct the Morse indices on all curve segments of $\Sigma_{\mathrm{abs}}^{*}$ between triple points and double roots by computing all $2 N$ solutions of $\mathcal{A}(\lambda, v ; \gamma)=0$ at all double roots and triple points (or at arbitrary test points on each segment). Alternatively, we could compute all $2 N$ solutions $v_{j}$ of $\mathcal{D}\left(\lambda, v_{j}\right) u_{j}=0$ and (4.8) and (4.9) simultaneously, though this is computationally much more expensive.

We remark that it is not necessary to use the regularized system (4.8) and (4.9) away from double roots. Instead, it may be convenient for the implementation to use $\mathcal{D}(\lambda, v) u_{1}=0$ and $\mathcal{D}(\lambda, v+\mathrm{i} \gamma) u_{2}=0$.

Except for the location of double roots, these remarks apply equally to periodic coefficients and (4.10)-(4.12). For the sake of consistency with this case, we describe the set-up in AUTO for (4.8) and (4.9) as a first-order system and boundary-value problem, so there are $2 N+2 N$ complex equations. This way the same equation file of AUTO can be used. 


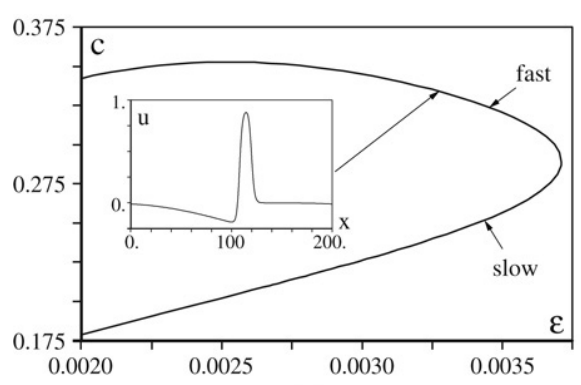

(a)

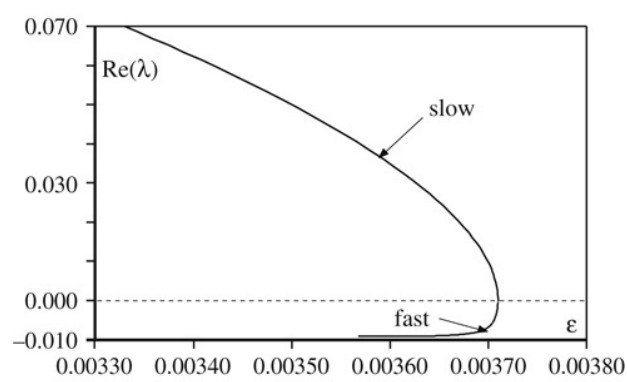

(b)

Fig. 2. (a) Bifurcation diagram of wave trains with period $L=200$ to the FitzHugh-Nagumo equation in the $(\epsilon, c)$ parameter plane. The inset shows the $u$-component of the fast wave train for $\epsilon=0.0033$. (b) The real part of the eigenvalue of $\mathcal{L}_{0}$ that changes sign at the fold bifurcation is plotted as a function of $\epsilon$. Here the lower branch corresponds to the fast wave trains and the upper one to the slow wave trains.

The constants file: We cast both (4.8) and (4.10) as ndim=8N real ODEs with $b c n d=8 \mathrm{~N}$ periodic boundary conditions and $i$ cnd $=4$ real integral conditions for normalization. The nicp $=5$ free parameters are $\lambda, \nu \in \mathbb{C}$ and $\gamma \in \mathbb{R}$. It is useful for subsequent computations to view $\gamma=\operatorname{Im} \nu_{2}$ and include $\operatorname{Re} v_{2}$ in the implementation.

For the case of constant coefficients, eigenfunctions are spatially constant, so we set ntst $=1$ and ncol $=2$. It is useful to disable mesh adaptation by setting $i a d=0$ and to exclude the vector $v$ in (4.8) and (4.9) from the pseudo-arclength computation by $\mathrm{nthu}=4$ followed by $2 \mathrm{~N}$ lines of the form "〈component index $>0$ ".

The equations file: It is often useful to implement the operator $\mathcal{D}(\lambda, v)$ in a new subroutine that is called from the subroutine func, since (4.8) and (4.10) require two evaluations of $\mathcal{D}$. This makes it also easy to simultaneously continue all eigenvalues and thereby the Morse index, if feasible. The current Morse index can then be stored in an additional parameter. Boundary and integral conditions are implemented in the subroutine bncd and $i$ cnd as described previously in Section 3.5.

Initial data: For constant coefficients, we use double roots as described above and set the data in the subroutine stpnt. For periodic coefficients, initial points in the generalized absolute spectrum are often found by continuing single Floquet exponents in exponential weights to a point where two of these have the same real part. To improve convergence of the initialization, we recommend to join both eigenfunctions and the nonlinear solution into a single data file and rescale to the same discretization grid. The program @f c converts such a file to AUTO format and reads initial parameters from the subroutine stpnt, see [4].

\section{Examples}

To illustrate the algorithms outlined above, we investigate essential and absolute spectra for the FitzHugh-Nagumo and the complex Ginzburg-Landau equation. ${ }^{1}$

\footnotetext{
${ }^{1}$ The AUTO files used for the following computations are available from the authors upon request.
}

\subsection{The FitzHugh-Nagumo equation}

Our first example is concerned with the classical FitzHughNagumo equation

$$
\begin{aligned}
& u_{t}=u_{x x}+c u_{x}-v-u(u-1)(u-a) \\
& v_{t}=\delta v_{x x}+c v_{x}+\epsilon(u-\gamma v),
\end{aligned}
$$

one of the best understood models for excitable media. Here, we already passed to a frame moving with speed $c$. Our goal is to investigate the critical part of the spectrum of wave trains $(u, v)(x)=(u, v)(x+2 \pi / k)$.

It is known, from numerical computations and through theoretical work, that the FitzHugh-Nagumo equation supports, in an appropriate parameter regime, a fast stable pulse and an unstable slow pulse which disappear in fold or saddlenode bifurcations as the parameter $\epsilon$ is increased. Both pulses are accompanied by wave trains with arbitrarily large spatial period, which converge to the pulses as the period is increased and also undergo saddle-node bifurcations for each fixed period as $\epsilon$ is increased. Our objective is to numerically continue the spectrum of these wave trains, which will cross the imaginary axis as we continue the wave trains for a fixed large period through their fold bifurcation. For large periods, the eigenvalues of the pulses generate nearby isolas of essential spectrum [5] and so we expect an isola to cross at the fold point. Throughout, we fix the parameters $a=\gamma=0.2$ and $\delta=0.25$, and consider the wave trains with spatial period $L=200$. The bifurcation diagram in $(c, \epsilon)$ and the associated solution profiles are shown in Fig. 2(a).

First, to illustrate the PDE spectra near the fold bifurcation, we continue the fast wave trains in the $(\epsilon, c)$-plane until they become the slow wave trains while, at the same time, computing and continuing the simple real eigenvalue of their PDE linearization $\mathcal{L}_{0}$ that destabilizes the wave train at the fold. The resulting eigenvalue curve is shown in Fig. 2(b).

Next, we compute the isolas of essential spectrum that emanate from the fold eigenvalue and from the translation eigenvalue at $\lambda=0$ for different values of $\epsilon$ near the fold bifurcation. As illustrated in Fig. 3, the fast wave train destabilizes already before the actually fold bifurcation as the two aforementioned isolas first coalesce at the temporal eigenvalues corresponding to $v=\mathrm{i} \pi / L$ to form a single isola, part of which then moves into the right half-plane. Note 


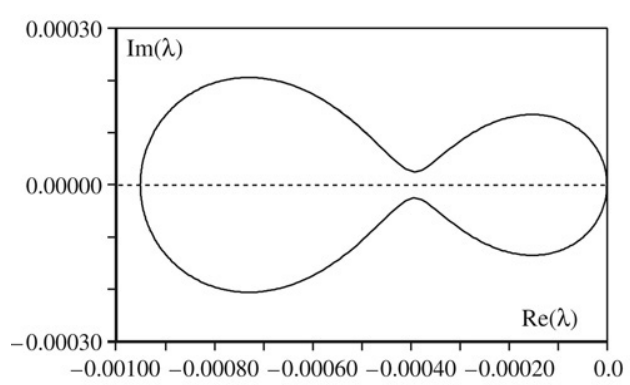

(a)

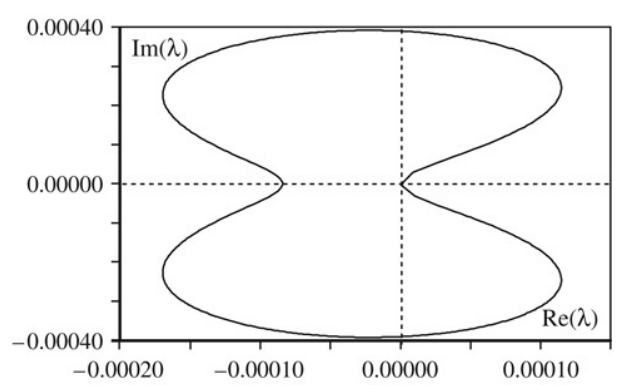

(b)

Fig. 3. (a) $\epsilon \approx 0.00371$ : The isola corresponding to the fold eigenvalue has merged with the isola at the origin. (b) $\epsilon \approx 0.00371013$ : The merged isolas of the fast wave train have already crossed the imaginary axis before the fold point.

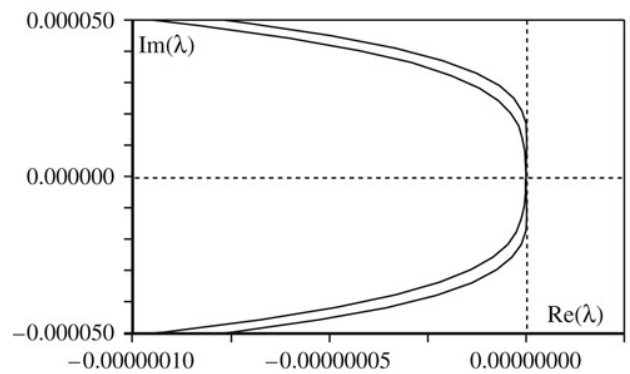

(a)

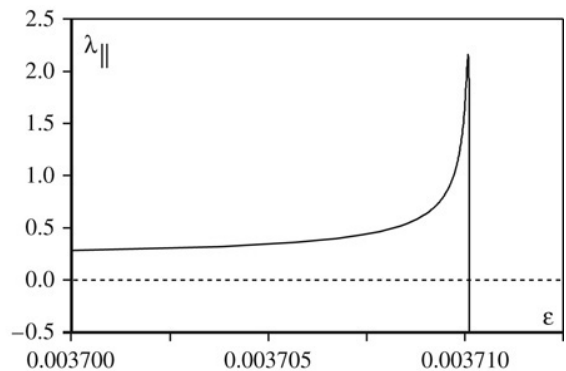

(b)

Fig. 4. Details of the onset of instability of the isola shown in Fig. 3. (a) We plot an overlay of the critical parts of the isola for $\epsilon \approx 0.00371011259$ and $\epsilon \approx 0.00371011266$. (b) We plot the tangency coefficient $\lambda_{\|}$(see Section 3.4.3) as a function of $\epsilon$, which corroborates that the onset occurs at zero wavenumber.

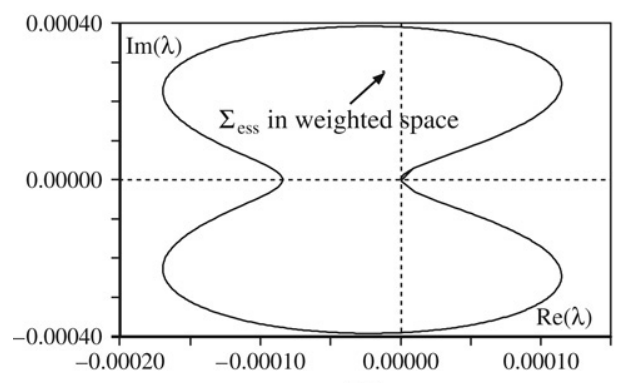

(a)

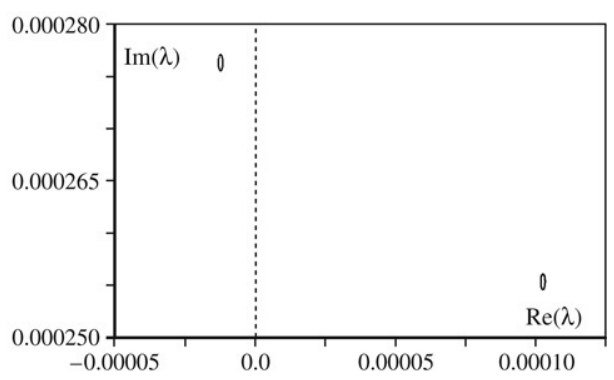

(b)

Fig. 5. (a) The isola from Fig. 3(b) in the unweighted space and the upper part of the same isola, now computed in a weighted space with Re $v \approx-0.035$. (b) Magnification of the isola in the weighted space for two different values of $\epsilon$ : the unstable isola to the right corresponds to a value of $\epsilon$ closer to the fold. Both isolas contain absolute spectrum, cf. [10].

that the unfolding of the essential spectrum near the temporal double root that occurs when the two isolas touch each other is the $\mathrm{x}$-shape crossing that we expect from the list of generic singularities in Section 3.3.3. Fig. 4 indicates that the onset of instability does not occur at finite wavenumbers; instead the curvature of the essential spectrum at the origin changes sign, see Section 3.4.3. We remark that at the fold point the isola has an $\mathrm{x}$-shaped crossing point at the origin and the group velocity changes sign through a singularity.

The merged isola in Fig. 3(b) contains absolute spectrum, which we found hard to compute directly though. Instead, we located it indirectly via isolas of essential spectrum, computed in exponentially weighted spaces, which necessarily contain absolute spectrum of index 2 on account of the discussion in Section 4.3. Fig. 5 shows these isolas inside the isola plotted in Fig. 3(b). The isola containing absolute spectrum moves into the unstable half-plane as the parameter $\epsilon$ approaches the fold point (see Fig. 5(b)). Thus, the wave train is not only essentially but also absolutely unstable before the fold point.

Lastly, on the branch corresponding to the slow wave train, the merged isolas separate again into an unstable isola which is completely contained in the right half-plane and an isola which emerges from $\lambda=0$ and is contained in the closed right half-plane (see Fig. 6). Again by Section 4.3, both of these isola contain absolute spectrum. Concerning the isola attached to the origin, we located a point in the absolute spectrum by continuation of two Floquet exponents whose imaginary parts differ by $\pi / L$ and hence have opposite signs as Floquet multipliers, see Fig. 7. The expected curve of absolute spectrum containing this point seems hard to compute. However, since the signs of the real Floquet multipliers $\mathrm{e}^{\nu_{2}}$ and $\mathrm{e}^{\nu_{3}}$ are opposite, the crossing point of the real parts of the Floquet exponents is 


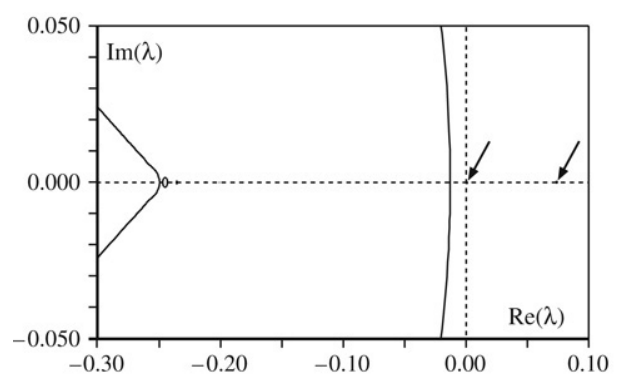

(a)

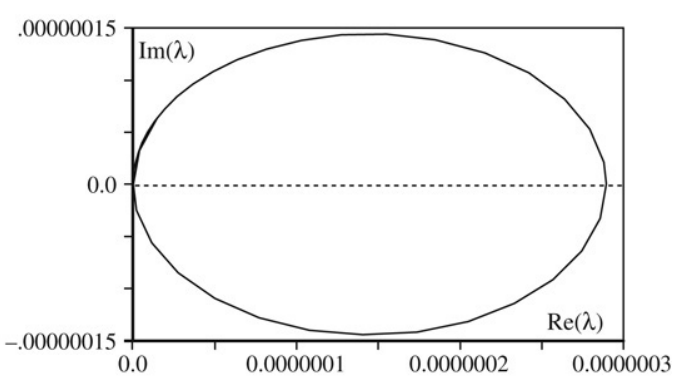

(b)

Fig. 6. (a) The essential spectrum of the slow periodic wave train at $\epsilon=0.0033$ near the origin is plotted. The two tiny isolas located near $\lambda=0$ and near $\lambda=0.08$ have been marked to be visible. (b) Magnification of the isola which is attached to the origin.

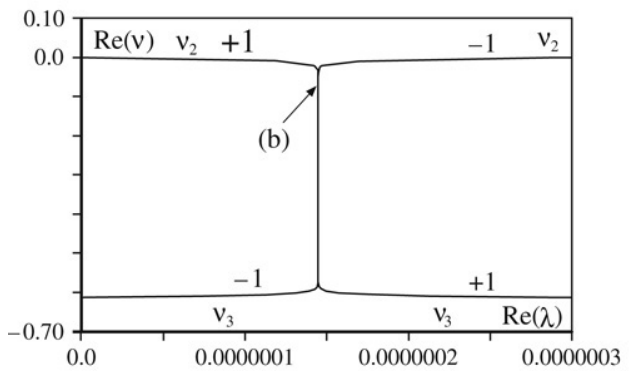

(a)

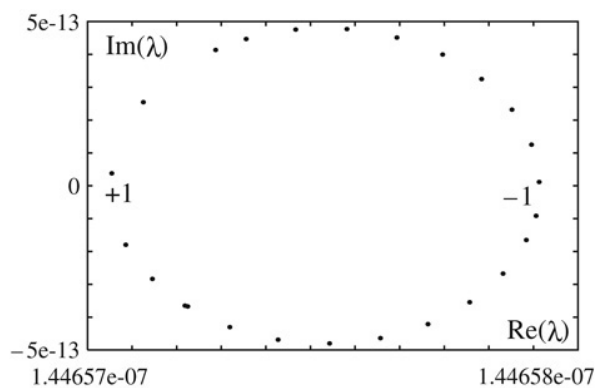

(b)

Fig. 7. (a) Real parts of the ordered Floquet exponents $v_{2}$ and $\nu_{3}$ for $\lambda \in \mathbb{R}$ within the isola of essential spectrum in Fig. $6(\mathrm{~b})$. Here, $\operatorname{sgn}\left(\mathrm{e}^{\nu_{2} L}\right)=-\operatorname{sgn}\left(\mathrm{e}^{\nu_{3} L}\right)$ with signs as indicated. The crossing point $\lambda \approx 1.446575 \times 10^{-7}$ lies in the absolute spectrum. (b) We plot the isola of essential spectrum in Fig. 6 (b) continued to the exponential weight $\eta \approx-0.075$. Signs of real Floquet multipliers are indicated. This isola contains the component of the absolute spectrum referred to in (a).

not a double root and the attached curve of absolute spectrum should cross the real axis with orthogonal tangent. We bound the location of this curve of absolute spectrum by continuing the isola of essential spectrum in Fig. 6(b) in decreasing exponential weight $\eta=\operatorname{Re} v \leq 0$. These isolas in weighted spaces appear to be concentric circles about the crossing point, and the component of absolute spectrum lies inside the smallest isola we computed, a circle of radius $5 \times 10^{-13}$.

\subsection{The complex Ginzburg-Landau equation}

Our second example exemplifies how the methods can be adapted outside of the class of reaction-diffusion systems, allowing for a complex diffusion coefficient with positive real part. We consider wave trains of the complex Ginzburg-Landau equation (CGL)

$A_{t}=(1+\mathrm{i} \alpha) A_{x x}+A-(1+\mathrm{i} \beta) A|A|^{2}$,

which is an approximate modulation equation valid near the onset of certain instabilities of the essential spectrum; see the review [8] and references therein.

Periodic wave train solutions of (5.1) are given by $A_{*}=$ $r \mathrm{e}^{\mathrm{i}(\kappa x-\omega t)}$ with $r^{2}=1-\kappa^{2}$ and $\omega=\beta+(\alpha-\beta) \kappa^{2}$. In the detuned variable $A=\tilde{A} \mathrm{e}^{-\mathrm{i} \omega t}$, the equation becomes, upon omitting tildes,

$A_{t}=(1+\mathrm{i} \alpha) A_{x x}+(1+\mathrm{i} \omega) A-(1+\mathrm{i} \beta)|A|^{2} A$

with solutions $A_{*}=r \mathrm{e}^{\mathrm{i} \kappa x}$. For the linearization about these wave trains, we consider $B$ and $\bar{B}$ as independent variables, not necessarily complex conjugate, and obtain the linearization

$\lambda B=(1+\mathrm{i} \alpha) B_{x x}+(1+\mathrm{i} \omega) B-(1+\mathrm{i} \beta)\left(2\left|A_{*}\right|^{2} B+A_{*}^{2} \bar{B}\right)$

$\lambda \bar{B}=(1-\mathrm{i} \alpha) \bar{B}_{x x}+(1+\mathrm{i} \omega) \bar{B}-(1-\mathrm{i} \beta)\left(2\left|A_{*}\right|^{2} \bar{B}+\bar{A}_{*}^{2} B\right)$.

Next, we substitute $B=b \mathrm{e}^{\mathrm{i} \kappa x+v x}$ and $\bar{B}=\bar{b} \mathrm{e}^{-\mathrm{i} \kappa x+v x}$, where we view $b$ and $\bar{b}$ as independent variables. We obtain the analogue to the matrix in (4.11) for the dispersion relation

$$
\begin{aligned}
\mathcal{D}(\lambda, \nu)= & \left(\begin{array}{cc}
(1+\mathrm{i} \alpha)(\nu+\mathrm{i} \kappa)^{2} & 0 \\
0 & (1-\mathrm{i} \alpha)(\nu-\mathrm{i} \kappa)^{2}
\end{array}\right) \\
& +a-\lambda \mathrm{i} d
\end{aligned}
$$

where

$a=\left(\begin{array}{cc}1+\mathrm{i} \omega-2(1+\mathrm{i} \beta) r^{2} & -(1+\mathrm{i} \beta) r^{2} \\ -(1-\mathrm{i} \beta) r^{2} & 1-\mathrm{i} \omega-2(1-\mathrm{i} \beta) r^{2}\end{array}\right)$

which simplifies to the equation in Box I. Hence, we obtain a constant-coefficient problem with the dispersion relation $d(\lambda, \nu)=\operatorname{det} \mathcal{D}(\lambda, \nu)=0$, and the approach of Section 3.3 and Section 4.2 for $N=2$ applies.

The essential spectrum $\{\lambda ; d(\lambda, \mathrm{i} \gamma)=0, \gamma \in \mathbb{R}\}$ consists of the two explicit curves as given in Box II.

We note that $\lambda_{-}(0)=0$, so zero is always in the essential spectrum (see Fig. 9(a) for the shape of the essential spectrum).

Regarding the absolute spectrum, note that the dispersion relation $d$ has four spatial roots $v$ for each $\lambda$, and the Morse index for the absolute spectrum is therefore two. Furthermore, we expect $\left(\begin{array}{l}4 \\ 2\end{array}\right)=6$ double roots by Lemma 4.5 ; note, however, that this prediction will not hold for $\alpha=0$ since the diffusion 


$$
\mathcal{D}(\lambda, \nu)=-\lambda \mathrm{i} d+\left(\begin{array}{cc}
(1+\mathrm{i} \alpha)\left(v^{2}+2 \mathrm{i} \kappa \nu\right)-(1+\mathrm{i} \beta) r^{2} & -(1+\mathrm{i} \beta) r^{2} \\
-(1+\mathrm{i} \beta) r^{2} & (1-\mathrm{i} \alpha)\left(v^{2}-2 \mathrm{i} \kappa \nu\right)-(1-\mathrm{i} \beta) r^{2}
\end{array}\right)
$$

Box I.

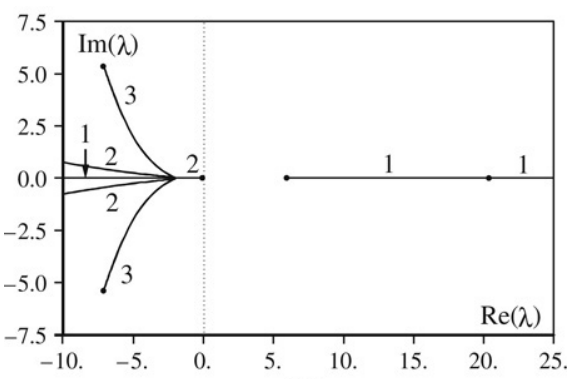

(a)

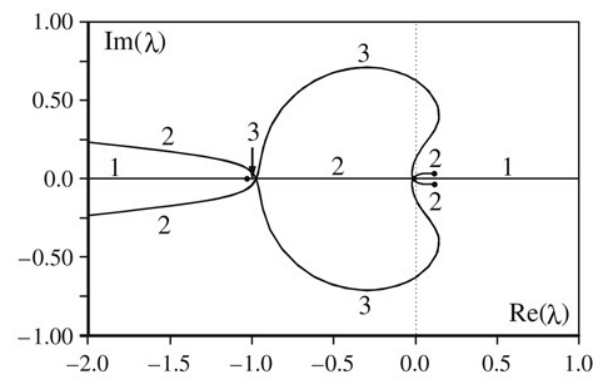

(b)

Fig. 8. The generalized absolute spectrum $\Sigma_{\text {abs }}^{*}$ is plotted for $(\alpha, \beta)=(0.1,0.2)$, where bullets correspond to branch points and numbers indicate the Morse index. (a) For the wavenumber $\kappa=0.1$ the absolute spectrum, $\Sigma_{\text {abs }}^{2}$, is stable, and its rightmost point is a branch point at $\lambda \approx-0.0001$. (b) For the wavenumber $\kappa=0.7$ the absolute spectrum is unstable, and its rightmost points are branch points at $\lambda \approx 0.115 \pm 0.036 \mathrm{i}$ (here we omitted two branch points of index one at $\lambda \approx 189$ and $\lambda \approx 210$ ).

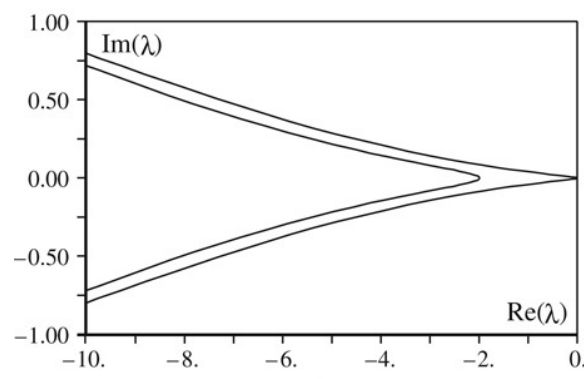

(a)

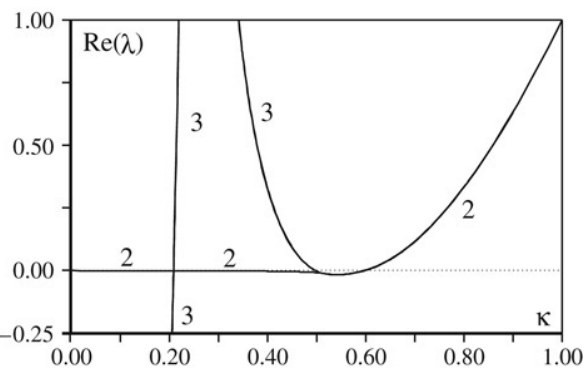

(b)

Fig. 9. (a) The essential spectrum of the wave train with wavenumber $\kappa=0.1$ is plotted for $(\alpha, \beta)=(0.1,0.2)$. (b) For $(\alpha, \beta)=(0.1,0.2)$, we plot the real parts of branch points with different Morse indices as functions of $\kappa$. The absolute spectrum becomes unstable at $\kappa \approx 0.598$ through branch points at $\lambda \approx \pm 0.032 \mathrm{i}$ of index two.

$$
\lambda_{ \pm}(\mathrm{i} \gamma)=\kappa^{2}-1-\gamma(2 \mathrm{i} \alpha \kappa+\gamma) \pm \sqrt{\left(\kappa^{2}-1\right)^{2}-\gamma\left(4 \mathrm{i} \beta \kappa^{3}+2 \alpha \beta \gamma+\alpha^{2} \gamma^{3}-4 \mathrm{i} \kappa\left(\beta+\alpha \gamma^{2}\right)-2 \kappa^{2}(2+\alpha \beta) \gamma\right)}
$$

Box II.

coefficients coincide in this case, and Lemma 4.5 does not apply. Indeed, the resultant of $d(\lambda, v)$ and $\partial_{\nu} d(\lambda, \nu)$ with respect to $v$ has degree four in that case, hence there are only four double roots (plus two at infinity). We now discuss the set $\Sigma_{\text {abs }}^{*}$ for various different parameter values.

We focus on the complex Ginzburg-Landau equation with $\alpha \neq 0$ for which essential and absolute spectrum generally differ. Furthermore, the explicit solution (Box II) is not easy to interpret for general $\alpha, \beta$ and $\kappa$. Therefore, it appears appropriate to use the numerical approaches discussed in Section 4.2 to compute the absolute spectrum.

Our results are summarized in Figs. 8 and 10, where we plot the numerically computed sets $\Sigma_{\mathrm{abs}}^{*}$ and the indices associated with each segment for three sets of parameter values. The union of the segments with index 2 is the absolute spectrum. Of interest is the onset of absolute instability, which we computed for fixed values of $(\alpha, \beta)$ as the wavenumber $\kappa$ is varied. For $(\alpha, \beta)=(0.1,0.2)$, the absolute spectrum becomes unstable through a complex conjugate pair of branch points that crosses the imaginary axis, while for $(\alpha, \beta)=(-8,1)$ all branch points lie to the left of the imaginary axis, and the instability is induced by a pair of complex conjugate curves of absolute spectrum that crosses the imaginary axis.

First, consider $(\alpha, \beta)=(0.1,0.2)$. Starting with the stable absolute spectrum for $\kappa=0.1$ shown in Fig. 8(a), we continued the branch points in the parameter $\kappa \in[0,1]$ to locate the onset of absolute instability (see Fig. 9(b)). The real stable branch point in the absolute spectrum for $\kappa=0.1$ shown in Fig. 8 (a) merges with a branch point of index 3 at $\kappa \approx 0.51$ and $\operatorname{Re} \lambda \approx$ -0.01 . For increasing $\kappa$ a pair of complex conjugate branch points emerges, each with index 2 , and crosses the imaginary axis at $\kappa \approx 0.598$. This is the only crossing of branch points in the absolute spectrum for $(\alpha, \beta)=(0.1,0.2)$ and $\kappa \in[0,1]$. 


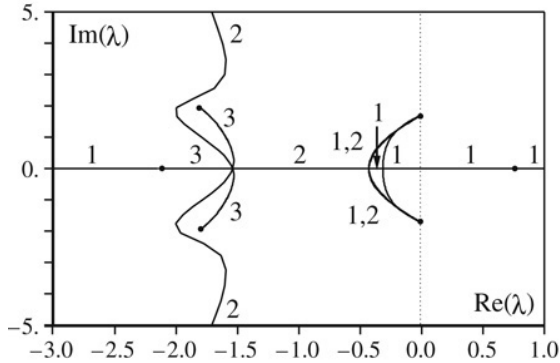

(a)

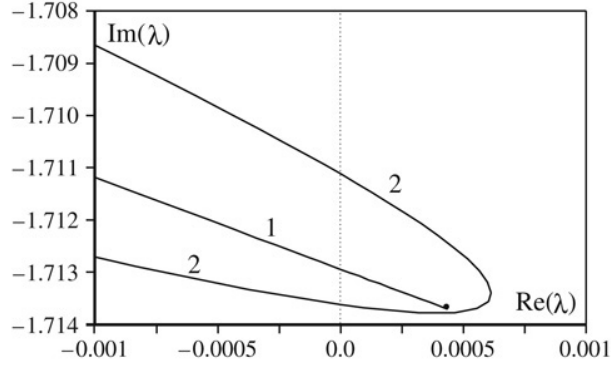

(b)

Fig. 10. (a) The absolute spectrum is plotted for $(\alpha, \beta)=(-8,1)$ and $\kappa=-0.3$. Note that $\Sigma_{\text {abs }}^{2}$ is unstable, but that there are no branch points with index two. (b) Magnification of one of the critical regions.

Next, we consider the Ginzburg-Landau equation for $(\alpha, \beta)=(-8,1)$, which lie in the Benjamin-Feir unstable regime $\alpha \beta<1$, and focus on the wave train with wavenumber $\kappa=-0.3$. The generalized absolute spectrum is plotted in Fig. 10. In this case, the absolute spectrum is unstable but does not contain any branch points. In particular, the instability is a remnant instability (in the terminology of [13]) which cannot be detected by locating branch points of index two.

\section{Acknowledgments}

J. Rademacher acknowledges hospitality at the Free University of Berlin and support from NSF grant DMS-0203301, a PIMS fellowship and DFG priority program SPP 1095. B. Sandstede acknowledges support from the NSF through grant DMS-0203854 and from a Royal Society Wolfson Research Merit Award. A. Scheel was partially supported by the NSF through grants DMS-0203301 and DMS-0504271.

\section{References}

[1] R.J. Briggs, Electron-Stream Interaction with Plasmas, MIT Press, Cambridge, 1964.

[2] E. Crampin, Reaction-diffusion patterns on growing domains. Ph.D. Thesis, University of Oxford, 2000.

[3] M.C. Cross, P.C. Hohenberg, Pattern formation outside of equilibrium, Rev. Modern Phys. 65 (1993) 851-1112.

[4] E. Doedel, R.C. Paffenroth, A.R. Champneys, T.F. Fairgrieve, Y.A. Kuznetsov, B.E. Oldeman, B. Sandstede, X. Wang, AUTO2000: Continuation and bifurcation software for ordinary differential equations (with HOMCONT), Technical Report, Concordia University, 2002.

[5] R.A. Gardner, On the structure of the spectra of periodic travelling waves, J. Math. Pures Appl. 72 (1993) 415-439.

[6] D. Henry, Geometric theory of semilinear parabolic equations, in: Lecture Notes in Mathematics, vol. 840, Springer, Berlin, 1981.
[7] M. Heroux, R. Bartlett, V. Howle, R. Hoekstra, J. Hu, T. Kolda, R. Lehoucq, K. Long, R. Pawlowski, E. Phipps, A. Salinger, H. Thornquist, R. Tuminaro, J. Willenbring, A. Williams, An overview of Trilinos, Technical Report SAND2003-2952, Sandia National Laboratories, 2003.

[8] A. Mielke, The Ginzburg-Landau equation in its role as a modulation equation, in: B. Fiedler (Ed.), Handbook of Dynamical Systems II, Elsevier, 2002, pp. 759-834.

[9] J.D.M. Rademacher, Homoclinic bifurcation from heteroclinic cycles with periodic orbits and tracefiring of pulses, Ph.D. Thesis, University of Minnesota, 2004.

[10] J.D.M. Rademacher, Geometric relations of absolute and essential spectra of wave trains, SIAM J. Appl. Dyn. Syst. 5 (2006) 634-649.

[11] J.D.M. Rademacher, R.W. Wittenberg, Viscous shocks in the destabilized Kuramoto-Sivashinsky equation, J. Comput. Nonlinear Dyn. 1 (2006) 336-347.

[12] B. Sandstede, A. Scheel, Absolute versus convective instability of spiral waves, Phys. Rev. E 62 (2000) 7708-7714.

[13] B. Sandstede, A. Scheel, Absolute and convective instabilities of waves on unbounded and large bounded domains, Physica D 145 (2000) 233-277.

[14] B. Sandstede, A. Scheel, On the structure of spectra of modulated travelling waves, Math. Nachr. 232 (2001) 39-93.

[15] B. Sandstede, A. Scheel, Gluing unstable fronts and backs together can produce stable pulses, Nonlinearity 13 (2000) 1465-1482.

[16] B. Scarpellini, $L^{2}$-perturbations of periodic equilibria of reaction-diffusion systems, Nonlinear Differ. Equ. Appl. 1 (1994) 281-311.

[17] S.A. Suslov, Numerical aspects of searching convective/absolute instability transition, J. Comput. Phys. 212 (2006) 188-217.

[18] L.N. Trefethen, M. Embree, Spectra and Pseudospectra, Princeton University Press, Princeton, 2005.

[19] P. Wheeler, D. Barkley, Computation of spiral spectra, SIAM J. Appl. Dyn. Syst. 5 (2006) 157-177.

[20] M.G. Zimmermann, S.O. Firle, M.A. Natiello, M. Hildebrand, M. Eiswirth, M. Bär, A.K. Bangia, I.G. Kevrekidis, Pulse bifurcation and transition to spatiotemporal chaos in an excitable reaction-diffusion model, Physica D 110 (1997) 92-104. 